\title{
Meeting Abstracts of the BACR conference: response and resistance in cancer therapy
}

\author{
Canterbury, UK; 10-12 Sep 2018; Published: 19 Dec 2018
}

Correspondence to: Tim R. Fenton, Michelle D. Garrett, Martin Michaelis, Mark N. Wass, School of Biosciences, University of Kent, Canterbury CT2 7NJ, UK. E-mails: t.fenton@kent.ac.uk; m.d.garrett@kent.ac.uk; m.michaelis@kent.ac.uk; m.n.wass@kent.ac.uk

\section{Targeting chemoresistance induced by $M Y C N$ and $A L K$ in neuroblastoma}

\section{Louis Chesler}

Institute of Cancer Research, London, UK

Neuroblastoma, a tumour of peripheral nerve, is the most common solid tumour of young children and in high-risk diseases, which comprises approximately half of patients, and the death from chemoresistant, metastatic relapse is very frequent. Children who relapse exhibit clonal enrichment of two genomic alterations: high-level amplification of the MYCN oncogene, and kinase domain mutations of the anaplastic lymphoma kinase $(A L K)$ gene. Overall survival in this patient cohort is $<15 \%$ at 3 years, and there are few options for rationally targeted therapy. Neuroblastoma patients exhibit de novo resistance to existing ALK inhibitors, and no clinical therapeutics to target $M Y C N$ has yet been developed. This talk will outline our efforts to model aberrant expression of $M Y C N$ and $A L K$ in neuroblastoma, and to uncover mechanisms of oncogenic action that are therapeutically targetable using small-molecule inhibitors. We describe a mechanistic interaction in which $A L K$ upregulates $M Y C N$ transcription, and discuss clinical trials emerging from our work to develop transcriptional inhibitors of $M Y C N$, and to identify effective inhibitors of ALK in neuroblastoma patients.

\section{Clinical application of single cell analysis}

\section{Bernhard Polzer}

Fraunhofer Item, Regensburg, Germany

Currently, one in four deaths is caused by cancer, mainly as a result of systemic spread and metastatic disease. Despite new drugs, the currently available therapies are effective only in one in four cancer patients. An initially successful therapy is often followed by a relapse of the disease after a few months only, which can be explained by the following two underlying mechanisms: on the one hand, due to the genetic hetero-

(C) The Author(s) 2018. Open Access This article is licensed under a Creative Commons Attribution 4.0 International License (https://creativecommons.org/licenses/by/4.0/), which permits unrestricted use, sharing, adaptation, distribution and reproduction in any medium or format, for any purpose, even commercially, as long as you give appropriate credit to the original author(s) and the source, provide a link to the Creative Commons license, and indicate if changes were made.

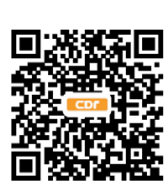


geneity found in many types of cancer, it is assumed that resistant tumor cells are already present in many patients at the time of therapeutic intervention; on the other hand, the administered drugs result in a selection of tumor cell clones that have developed de novo mutations favorable for tumor cell survival. Monitoring of genetic alterations in the course of systemic cancer disease at close intervals could thus help in recognizing the development of resistance at an early stage and in selecting the most appropriate therapy for each patient. For this, we established a liquid biopsy platform and single cell technologies to follow the genetic evolution of systemic cancer.

\title{
3. The resistant cancer cell line collection: drug-adapted cancer cell lines as preclinical models of acquired drug resistance
}

\author{
Martin Michaelis ${ }^{1}$, Mark N. Wass ${ }^{1}$, Cinatl J. Jr ${ }^{2}$ \\ ${ }^{1}$ Industrial Biotechnology Centre and School of Biosciences, University of Kent, Canterbury, UK \\ ${ }^{2}$ Institut für Medizinische Virologie, Klinikum der Goethe-Universität, Frankfurt am Main, Germany
}

Acquired resistance formation is a major reason for the failure of anti-cancer therapies. Pre-clinical model systems are needed to complement data derived from clinical specimens such as liquid biopsies to establish the understanding of the resistance formation process required to improve therapies. Cancer cell lines enable the generation of the large number of model systems needed to cover the complexity of the resistance mechanisms. Major resistance mechanisms have been discovered in drug-adapted cell lines, e.g., high levels of the ATP-binding cassette (ABC) transporters ABCB1 (also P-glycoprotein, MDR1) and ABCC1 (also MRP1). Moreover, various groups have used drug-adapted cancer cell lines to identify and elucidate clinically relevant acquired resistance mechanisms to targeted and cytotoxic anti-cancer drugs. We have established the resistant cancer cell line (RCCL) collection by adapting initially chemosensitive cancer cell lines to clinical concentrations of targeted and cytotoxic anti-cancer drugs. It currently contains 1,300 cancer cell lines based on 125 parental cell lines from 15 cancer entities and reflects acquired resistance to 67 drugs (https://research.kent.ac.uk/ibc/the-resistant-cancer-cell-line-rccl-collection). Using RCCL collection cell lines, we and others identified (clinically relevant) drug resistance mechanisms and have gained insights into the action of anti-cancer drugs. For example, we have shown that acquisition of resistance to MDM2 inhibitors is associated with TP53 mutations, which has been clinically confirmed. Furthermore, we have identified high SAMHD1 expression as a resistance mechanism in cytarabine-adapted acute myeloid leukaemia cell lines and, in turn, shown that SAMHD1 is a biomarker for cytarabine response in the clinic and a novel therapeutic target in acute myeloid leukaemia. In conclusion, drug-adapted cancer cell lines reflect clinically relevant acquired drug resistance mechanisms. The RCCL collection is a readily available tool containing a substantial number of preclinical models of acquired drug resistance in cancer.

\section{Prospects and limitations of patient-derived xenografts in translational cancer research}

\section{Iduna Fichtner ${ }^{1,2}$}

\author{
${ }^{1}$ Max Delbrück Center, Berlin, Germany \\ ${ }^{2}$ Experimental Pharmacology and Oncology GmbH, Berlin, Germany
}

The molecular analysis of tumours by highly sophisticated gene expression and sequencing methods allows the comprehensive identification of drugable targets and the increasing chance for personalized treatments. Clinically established examples are the use of Imatinib for $B C R-A B L$ positive leukemias and Vemurafenib 
for BRAF mutated melanomas. But, in general tumours are characterized by the presence of numerous oncogenes, genetic aberrations and a dynamic growth control. That complex and individual pattern even nowadays cannot fully be understood and mirrored by medical systems biology. Another attractive option to maintain the typical features of a patient tumour is by growing it in a foreign host how it is practised since the invention of the patient-derived xenografts (PDX). This method has been used for more than 30 years but got recently increasing attractivity for the identification of novel biomarkers and as so-called avatars for a patient-related optimization of therapy. Meanwhile, worldwide several thousands of PDX models covering the broad variety of tumour types exist and are used both for fundamental research and for commercial offer. It has been proved in several investigations that the PDX highly resemble the original tumour specimen concerning histology, molecular marker and response to therapy. Within the talk our own longstanding experiences concerning the establishment and characterization of PDX will be reflected. Additional efforts have been made to further "humanize" the mice. For that purpose, in general two procedures exist. A transient humanization can be reached by co-transplanting human mononuclear cells or subtypes of leukocytes like NK cells. A lifelong humanization of the mouse immune system can be reached by transplanting human stem cells. In these humanized mice immunopharmacological drugs like checkpoint inhibitors can be functionally tested. Methods and experiences with this sophisticated preclinical model system will be presented.

\section{Drug resistance to synthetic lethal treatments for cancer}

\section{Chris Lord}

\section{The Institute of Cancer Research, London, UK}

The first synthetic lethal treatments for cancer, poly (ADP-ribose) polymerase (PARP) inhibitors, are now approved for use. Despite PARP inhibitors delivering some sustained and profound clinical responses, drug resistance is a growing problem. I will discuss how genetic perturbation screens can identify mechanisms of PARP inhibitor resistance and how genetic reversions in BRCA genes, mutations in PARP1 and loss of the newly identified Shieldin protein complex can result in PARP inhibitor resistance. I will also discuss how the mechanism of PARP inhibitor resistance that emerges defines the sensitivity to other therapeutics.

\section{Assessment of homologous recombination DNA repair function in human tumours and potential for selection of patients for platinum or PARP inhibitor therapy}

\section{Nicola Curtin}

\section{Newcastle University, UK}

All cells experience a high level of base lesions and DNA single strand breaks (SSBs) caused by endogenously generated reactive oxygen species. Poly (ADP-ribose) polymerase (PARP) is crucial for the repair of SSBs and any left unrepaired will collapse replication forks, which can only be resolved by HRR. We first described the selective killing of cells defective in homologous recombination DNA repair (HRR) by PARP is in 2005, which has led to an explosion in PARP inhibitor (PARPi) research. BRCA1 and BRCA2, the breast and ovarian cancer genes are key components of HRR and 3 PARPi are approved for use in ovarian cancer. Since (1) $<20 \%$ of ovarian cancers are associated with BRCA mutations; (2) HRR is a multi-component pathway such that defects in any one of them may compromise HRR function; and (3) HRR dysfunction confers sensitivity to platinum agents and $60 \%$ of ovarian cancers respond to platinum therapy, we 
investigated HRR function in primary cultures of cells from ascites taken from patients with ovarian cancer. We measured HRR function by the ability to form RAD51 foci following $24 \mathrm{~h}$ exposure to rucaparib (a PARPi). We found that $55 \%$ of our samples were HRR dysfunctional (HRD), this was later reinforced by the estimate of $51 \%$ HRD by the TCGA, following an extensive genomic analysis. Subsequent work showed that those patients identified as HRR defective had better PFS and OS, indicating a better response to Standard of Care: carboplatin-paclitaxel therapy. We have applied this test to pleural effusions and found HRR defects were relatively common, including 3 out of 4 non-small-cell lung cancer samples. More recently we have investigated the HRR status of primary cultures of ascites cells taken from patients with a variety of different tumour types and found that $8 / 24$ were HRD. Survival of patients treated with platinum was greater in the HRD group $(n=3)$ but this was not significant due to the small number of patients (10 in total). Our data indicate that HRD is relatively common in a variety of tumour types and the limited data on their response to platinum therapy suggest that adoption of either platinum or PARPi therapy in patients with any HRD tumour is worthy of investigation.

\title{
7. Pharmaco Epigenetics in human cancer
}

\section{Manel Esteller}

\section{IDBELL, Barcelona, Spain}

For the last twenty-five years an increasing amount of evidence has shown the relevance of epigenetics in cell biology and tissue physiology, being DNA methylation aberrations in cancer the flag-ship for the recognition of its disturbance in human diseases. From the candidate gene approaches, new powerful technologies such as comprehensive DNA methylation microarrays and whole genome bisulfite sequencing have recently emerged that reinforce the notion of epigenetic disruption in the crossroad of many sickness. From the poster-boy cases of MGMT and GSTP1 hypermethylation in the prediction of alkylating drug response and prostate cancer detection, respectively, to the personalized treatment of leukemia with small molecules targeted to fusion proteins involving histone modifiers such as DOT1L and MLL, the field has walked a long path. The current talk will focus on the epigenetic profiling, basically at the level of DNA methylation and histone modifications, that is starting to provide clinical value in the diagnosis, prognosis and prediction of response to drug therapies, with an emphasis in neoplasia, but without forgetting the novel advances in other human disorders. For cancer, we have already a wide view of the undergoing DNA methylation events that expand beyond classical promoter CpG islands of tumor suppressor genes and we have a growing list of mutated chromatin remodeler genes that contributes to the tumorigenesis process. It is time to apply this knowledge in practical clinical situations like the diagnosis of cancers of unknown primary, the screening of malignancies in high-risk populations or a biomarker selection of the patients that should receive treatment with epigenetic drugs.

\section{A CRISPR-based mutagenesis method for determinants of drug resistance}

\section{Daniel J. O’Neill' ${ }^{1}$, Claudia Stellato ${ }^{1}$, Alexis Guernet ${ }^{1}$, Mike Firth ${ }^{1}$, Maryam Clausen ${ }^{2}$, Maria Emanuela Cuomo ${ }^{1}$}

\author{
${ }^{1}$ Discovery Sciences, IMED Biotech Unit, AstraZeneca, Cambridge, UK \\ ${ }^{2}$ Discovery Sciences, IMED Biotech Unit, AstraZeneca, Mölndal, Sweden
}

Acquired drug resistance represents a major challenge in the development of therapeutic agents that show long term effectiveness for the treatment of cancer. The problem is exemplified by non-small cell lung 
cancer (NSCLC) containing activating EGFR mutations. NSCLC tumours driven by EGFR variants are initially sensitive to tyrosine kinase inhibitors (TKIs), but some patients show renewed disease progression through expansion of tumour cell clones harbouring additional EGFR mutations. Models of drug resistance are therefore necessary to increase our understanding of resistance mechanisms, to predict variants that will arise in patients and to improve drug development strategies. CRISPR/Cas9 allows endogenous modification of genes and facilitates introduction of all possible mutations at a specific locus, whilst maintaining endogenous gene architecture, chromosomal context and epigenetic landscape. Here we describe a CRISPR-based methodology developed to identify "on target" mutations in key oncogenes that drive pharmacological resistance. Using EGFR as a target, we show how directed evolution of some residues in the EGFR kinase domain can be predictive of the resistance observed in the clinic in response to known TKIs, such as gefitinib and osimertinib. In fact, in the region analysed, we can recapitulate the variants observed clinically. We also identify amino acids chemically and structurally able to confer resistance to a TKI in structurally conserved tyrosine kinases such as BCR-Abl and Kit. The strategy also allows direct generation of disease relevant cellular models that recapitulate clinical drug resistance.

\section{Molecular data integration for response prediction}

\section{Lodewyk Wessels}

\section{Netherlands Cancer Institute, Amsterdam, Netherlands}

The exact mechanisms involved in tumor development and therapy response are still largely unclear. Here we report on two computational approaches to systematically unravel these mechanisms and show how these can be employed to predict response to anti-cancer agents. Clinical response to anti-cancer drugs varies between patients and is modulated by molecular features. Classic approaches to integrate these data for therapy response prediction almost exclusively employ gene expression data. Such predictors are difficult to interpret. We developed TANDEM, a two-stage approach in which the first stage explains response using upstream features (mutation, copy number and methylation) and the second stage explains the remainder using downstream features (gene expression). Applying TANDEM to 934 cell lines profiled across 265 drugs we show that the resulting models are more interpretable and equally predictive as classic approches. Second, following a more mechanistic integration approach, we constructed Bayesian models encompassing several of the important driver pathways and resistance mechanisms, and tested how well these models describe drug response data derived from a panel of breast cancer cell lines. The models provide estimates of the relative contribution of each of the drivers and resistance mechanisms and allow estimation of latent variables such as "pathway activation". We identify $4 \mathrm{EBP} 1$ protein expression as an important modulator of mTOR inhibitor response.

\section{Mechanisms of therapy resistance in prostate cancer}

\section{Charlotte Bevan}

\section{Imperial College London, UK}

Prostate tumours grow in response to androgens, which act via the androgen receptor (AR). Therapies for inoperable, disseminated disease, therefore, are largely designed to inhibit androgen signalling - via chemical castration (gonadal downregulation), inhibition of steroidogenesis and/or antiandrogens, which directly bind to and inhibit the action of the AR. These are initially successful in the vast majority of pa- 
tients, but almost inevitably tumours progress to an advanced castrate resistant stage [castration resistant prostate cancer (CRPC)]. Mechanisms of progression to CRPC are varied, but a common theme is that AR activity is retained and often amplified, despite very low (castrate) levels of circulating androgens. In a certain percentage of resistant tumours the AR itself is amplified, mutated or truncated, but this is not always the case. The AR is a member of the Nuclear receptor (NR) superfamily of transcription factors, and NRs share common cofactor proteins which act to increase or decrease their transcriptional activity - known as coactivators and corepressors, respectively. These cofactors are frequently multifunctional proteins, and AR cofactors include RNA splicing factors such as FUS, cytoplasmic chaperone and co-chaperone proteins such as p23 and BAG-1L, cell cycle and transcriptional effectors such as Prohibitin and some CDKs, the Hey proteins which are well-characterised Notch effectors, and tripartite motif proteins. We and others have shown that amplification of coactivators and loss or dysregulation of corepressors are also mechanisms of therapy resistance in prostate cancer. Interestingly we found that androgen treatment of prostate cancer cells results in downregulation of certain corepressors, thus the AR itself may promote tumour growth by downregulating its own repressors. Manipulation of AR cofactors is thus a valid therapeutic strategy for CRPC, and we have demonstrated that both inhibition of coactivators and increasing levels of corepressors can inhibit growth and invasion of prostate cancer cells, in vitro and in vivo. Other factors that can affect AR levels and activity are microRNAs, which usually act by reducing levels of their target mRNAs. They play vital roles in prostate cancer development, progression and metastasis and we hypothesised that microRNAs that modulate AR activity in lethal CRPC represent novel therapeutic targets for this disease. We used a high-throughput functional screen to systematically identify such microRNAs, and have shown that microRNAs can affect AR either directly or by targeting key cofactors, including the corepressor Prohibitin. Inhibitors of AR-modulatory microRNAs dramatically reduces AR activity and growth, migration and invasion of prostate cancer cells, and may thus represent novel prostate cancer therapeutics. They also show additive effects with AR silencing or antiandrogen treatment, suggesting potential combinatorial applications for prostate cancer treatment. A major advantage of microRNAs is that they are released from tumours into the circulation, where they can be detected and quantified. They thus also have enormous potential as prognostic and predictive biomarkers.

\section{Tyrosine Phosphorylation of nuclear PTEN in Glioma promotes therapeutic resistance through DNA damage repair}

\section{Frank Furnari}

Ludwig Institute for Cancer Research, University of California, San Diego, USA

Ionizing radiation (IR) and chemotherapy are standard of care treatments for glioblastoma (GBM) patients and both result in DNA damage, however their clinical efficacy is limited due to therapeutic resistance. Here, we identified a mechanism of such resistance mediated by nuclear PTEN phosphorylated on tyrosine 240 (pY240-PTEN) by FGFR2 kinase. pY240-PTEN is rapidly elevated and bound to chromatin in response to IR treatment and facilitates the recruitment of DNA repair protein, RAD51, to sites of damage. Thus, cells with high levels of pY240-PTEN are resistant to DNA damage and blocking Y240 phosphorylation with FGFR inhibitors confers radiation sensitivity to tumors and extends survival in GBM preclinical models. The central importance of PTEN Y240 phosphorylation in mediating DNA damage repair is further highlighted by the extreme radiation sensitivity of Y240F-PTEN knock-in mice. These results suggest that FGFR-mediated phosphorylation of PTEN Y240 is a key mechanism of radiation resistance and is an actionable target for improving radiotherapy efficacy in GBM patients. 


\section{Understanding and exploiting the hypoxia-induced DNA damage response}

\section{Ester Hammond}

\section{Oxford Institute for Radiation Oncology, UK}

Exposure to severe levels of hypoxia, sometime referred to as radiobiological hypoxia, leads to the induction of the DNA damage response. As cancer cells in radiobiological hypoxia are significantly more resistant to radiation-induced DNA damage they negatively impact radiotherapy response. The aim of our work is to investigate the biological response and adaption by cancer cells to hypoxia, with the ultimate aim of developing new therapeutic strategies though the identification of critical molecular targets. Recently, we have demonstrated that the hypoxia-induced DNA damage response is a result of replication stress, in part due to diminished ribonucleotide reductase activity in the absence of oxygen. Despite the presence of replication stress and a DNA damage response in hypoxia, we have not observed the accumulation of DNA damage. Both the sources of hypoxia-induced replication stress and potential strategies to target this for therapeutic gain will be discussed.

\section{Breaking down barriers to tumour immunity}

\section{Awen Gallimore \\ Cardiff University, UK}

Whilst certain immunotherapies can drive activation and expansion of tumour-specific $\mathrm{T}$ cells, most patients undergoing these therapies do not exhibit objective responses. A significant bottleneck may be the failure of activated lymphocytes to infiltrate solid tumours and/or immunosuppressive mechanisms within the tumour microenvironment. Foxp3+ regulatory T cells (Treg) often accumulate in solid tumours where they help create an immunosuppressive niche. Using a mouse model of carcinogen-induced fibrosarcomas, we have examined how Treg depletion impacts on tumour growth. Results of the study show that tumour regression after Treg-depletion is highly variable and observed in only a proportion of animals. Comparing the genotype and phenotype of tumours recovered from responder and non-responder mice reveals several key features of the tumour microenvironment that distinguish the two groups. These include the type of blood vessels present, the composition of the extracellular matrix and the number of tumour infiltrating lymphocytes. The reciprocal influences of these features will be discussed as well as the potential for manipulating the tumour microenvironment in order to maximise the success of $\mathrm{T}$ cell-based immunotherapies.

\section{Combination immunotherapies: aiming at the tumour microenvironment}

\section{Gareth Thomas}

\section{University of Southampton, UK}

Using next generation sequencing and bioinformatic analyses combined with multiplexed immunochemistry, we have characterised molecular features of the tumour microenvironment that are associated with effective/ineffective anti-cancer immune responses, characterising prognostic/predictive lymphocyte signatures and identifying immune evasion mechanisms that are common across different types of cancer. In vivo preclinical testing confirms that specific evasion mechanisms can be targeted to improve response to standard immunotherapies, and suggests strategies for combination immunotherapies based on immune classification of tumours. 


\title{
15. Chromosomal instability, tumour evolution and treatment resistance
}

\section{Geoff Macintyre}

\section{CRUK Cambridge Institute, Cambridge, UK}

Tumours with chromosomal instability exhibit increased intratumoral heterogeneity and poor prognosis. Genetically distinct clones enable a wider sampling of the fitness landscape, increasing the chance of rapid progression and treatment resistance. Here, I will present our recent work on identifying different types of chromosomal instability through copy number signature analysis. I will highlight the role that chromosomal instability plays in tumour evolution and the challenges faced trying to move mutational signature analysis into the clinic.

\section{Intra-tumour signalling and targeted therapy in melanoma}

\section{Claudia Wellbrock}

\section{University of Manchester, UK}

The BRAF-kinase and the MAPK-pathway are targets of current melanoma therapies, where they can induce fast responses with minimal adverse effects. Our recent work has demonstrated that intra-tumour signalling can have a major impact on the efficacy of MAP-kinase pathway targeting drugs. We discovered that the melanoma-specific transcription factor MITF confers cell-autonomous resistance to MAPK pathway therapy. Recent work by others has also demonstrated that MITF is an essential survival factor for drug-addicted acquired resistant melanomas. We found that during treatment with BRAF and MEK inhibitors, MITF expression is up-regulated in responding tumours and as direct consequence of effective inhibition of the MAPK pathway. This MITF up-regulation during treatment counteracts the killing-effect of the drugs, thus initiating a phase of tolerance to treatment which allows acquired resistance to establish. We identified the HIV-protease inhibitor nelfinavir as an inhibitor of the drug-induced MITF up-regulation. In line with this activity, nelfinavir profoundly improves responses to MAP-kinase pathway targeting drugs in $B R A F$ and NRAS mutant melanoma. One of the major challenges in tackling acquired resistance to MAPK inhibitors is the phenotype heterogeneity found in melanoma. As such melanomas contain subpopulations of cells that can be described as MITF ${ }^{\text {high }}$ and MITF $^{\text {low }}$. We had shown previously that MITFphenotype heterogeneity is of advantage for melanoma invasion, but we revealed that it also contributes to the establishment of highly resistant tumours. We identified a novel mechanism based on intra-tumour communications that maintains phenotype heterogeneity, and targeting this mechanism overcomes heterogeneity driven resistance in melanoma.

\section{The NF-kB regulated gene, Claspin, is potential biomarker for the response to Chk1 inhibitors}

\author{
Jill E. Hunter ${ }^{1}$, Jacqueline A. Butterworth ${ }^{1}$, Michelle D. Garrett ${ }^{2,3}$, Ian Collins ${ }^{3}$, Neil D. Perkins ${ }^{1}$ \\ ${ }^{1}$ Institute for Cell and Molecular Biosciences, Faculty of Medical Sciences, Newcastle University, Newcastle \\ Upon Tyne, Newcastle NE2 4HH, UK \\ ${ }^{2}$ School of Biosciences, University of Kent, Canterbury CT2 7NJ, UK \\ ${ }^{3}$ The Institute of Cancer Research, Sutton SM2 5NG, UK
}


Disruption of the DNA damage response, frequently seen as a consequence of genetic mutation, can lead to more rapid onset of tumorigenesis. However, inhibiting those pathways which are still intact provides a potential therapeutic strategy for targeting tumours that have become dependent on their activity. For this reason, inhibitors of ATR and Chk1 represent a potential new class of anti-cancer therapies, currently in clinical trials. Here, we describe a mechanism, involving the Chk1 targeted phosphosite Thr505 on the NF$\kappa \mathrm{B}$ subunit, RelA, and how mutation of this site results in earlier onset of MYC-driven lymphomagenesis in vivo. We describe a positive feedback loop in which Chk1 phosphorylation of RelA at T505, together with the NF- $\mathrm{B}$ B subunit c-Rel, drives the expression of the ATR checkpoint kinase regulator Claspin in response to DNA replication stress in cancer cells. This in turn is required for maintenance of Chk1 activity. Loss of a single allele of the Clspn gene in mice is sufficient to drive earlier tumorigenesis and low levels of CLSPN mRNA expression are associated with worse survival in some forms of human cancer. Importantly, disruption of this pathway leads to resistance of cells to treatment with Chk1 inhibitors, a result with implications for the potential use of these drugs as cancer therapies. Taken together, these data suggest that Claspin mRNA levels may be a potential biomarker for the response to novel Chk1 inhibitors in the clinic, and also a prognostic factor in some cancers.

\title{
18. Novel strategies to target RNA splicing to overcome androgen receptor splice variant-7 signalling in castration resistant prostate cancer
}

\section{A. Sharp" ${ }^{1 \#}$, J. C. Welti ${ }^{1 \#}$, W. Yuan ${ }^{1}$, A. Paschalis ${ }^{1}$, I. Figueiredo ${ }^{1}$, C. Bertan ${ }^{1}$, V. S. Gil ${ }^{1}$, D. N. Ro- drigues $^{1}$, E. Knight ${ }^{2}$, J. Ning ${ }^{2}$, J. Francis ${ }^{2}$, D. Dolling ${ }^{1}$, A. Neeb ${ }^{1}$, G. Boysen ${ }^{1}$, M. Crespo ${ }^{1}$, B. Al- Lazikani $^{3}$, S. Carreira1, J. Luo ${ }^{4}$, A. Swain ${ }^{2}$, S. R. Plymate ${ }^{5}$, J. S. de Bono ${ }^{1}$}

\author{
${ }^{1}$ Division of Clinical Studies, The Institute of Cancer Research, UK \\ ${ }^{2}$ Tumour Profiling Unit, The Institute of Cancer Research, UK \\ ${ }^{3}$ Cancer Research UK Cancer Therapeutics Unit, The Institute of Cancer Research, UK \\ ${ }^{4}$ Johns Hopkins University School of Medicine, USA \\ ${ }^{5}$ University of Washington, USA \\ "The two authors contributed equally to this Abstract
}

The constitutively active androgen receptor (AR) splice variant-7 (AR-V7) drives persistent AR signalling in castration resistant prostate cancer (CRPC). We demonstrate that $\mathrm{AR}-\mathrm{V} 7$ protein increases as castration resistance emerges and associates with resistance to endocrine therapies. In addition, AR-V7 does not correlate with full length AR protein or AR copy number, suggesting that aberrant RNA splicing is critical for AR splice variant (AR-SV) generation. Targeting RNA splicing is an attractive strategy to prevent AR-V7 generation and we demonstrate a novel role of bromodomain and extra-terminal (BET) family protein inhibitors in supressing RNA splicing. Our data show that BRD2, BRD3 and BRD4 RNA expression correlate with AR driven transcription in CRPC biopsies. Consistent with this, chemical BET inhibition (BETi), and BET family protein knockdown, reduced AR-V7 expression and AR signalling in prostate cancer models. In addition, we show BETi regulate RNA processing reducing alternative splicing and AR-V7 expression. Furthermore, BETi reduce growth of prostate cancer cells and patient derived organoids with known AR mutations, AR amplification and AR-V7 expression. Consistent with this, BETi, unlike enzalutamide, decreases persistent AR signalling and growth of a patient derived xenograft with $A R$ amplification and ARV7 expression. Despite these promising data, the pleotropic effects of BETi on cellular pathways and treatment related toxicities remain a concern. In light of this, we determined critical factors for BETi mediated AR-V7 regulation in CRPC. Interrogation of BETi treated RNA expression data and a focused splicing factor siRNA screen identified splicing factor B (anonymised; Sf-B) crucial for RNA splicing, AR-V7 generation and PC cell growth. In addition; Sf-B expression correlates with AR signalling and is associated with reduced clinical benefit from current endocrine therapies. Based on our results we propose that inhibition of Sf-B provides a novel approach to target AR-V7 and other AR-SVs in CRPC. 
19. Withdrawal of MEK1/2 inhibitor reverses acquired resistance driven by BRAF amplification but drives EMT and chemoresistance in cells with amplified KRAS

\author{
Matthew J. Sale ${ }^{1}$, Kathryn Balmanno ${ }^{1}$, Jayeta Saxena', Eiko Ozono ${ }^{1}$, Rebecca E. Mclntyre ${ }^{2}$, \\ Katarzyna Wojdyla ${ }^{3}$, Rebecca Gilley ${ }^{1}$, Anna Woroniuk ${ }^{1}$, Karen D. Howarth ${ }^{4}$, Gareth Hughes ${ }^{5}$, \\ Jonathan R. Dry ${ }^{6}$, Mark J. Arends ${ }^{7}$, David Oxley ${ }^{3}$, Susan Ashton ${ }^{8}$, David J. Adams ${ }^{2}$, Julio Saez- \\ Rodriguez $^{9}$, Paul D. Smith ${ }^{5}$, Simon J. Cook ${ }^{1}$
}

\begin{abstract}
${ }^{1}$ Signalling Programme, The Babraham Institute, Babraham Research Campus, Cambridge CB22 3AT, UK
${ }^{2}$ Experimental Cancer Genetics, Wellcome Sanger Institute, Wellcome Genome Campus, Hinxton, Cambridgeshire CB10 1SA, UK

${ }^{3}$ Proteomics Facility, The Babraham Institute, Babraham Research Campus, Cambridge CB22 3AT, UK

${ }^{4}$ Hutchison-MRC Research Centre, Department of Pathology, University of Cambridge, Hills Road, Cambridge $C B 2$ oXZ, UK

${ }^{5}$ Oncology Bioscience, Innovative Medicines and Early Development Biotech Unit, AstraZeneca, CRUK Cambridge Institute, Robinson Way, Cambridge CB2 oRE, UK

${ }^{6}$ Oncology Bioscience, Innovative Medicines and Early Development Biotech Unit, AstraZeneca, 35 Gatehouse Drive, Waltham, Massachusetts 02451, USA

${ }^{7}$ Division of Pathology, Centre for Comparative Pathology, Cancer Research UK Edinburgh Centre, MRC Institute of Genetics \& Molecular Medicine, University of Edinburgh, Western General Hospital, Crewe Road South, Edinburgh EH4 $2 X R$, UK

${ }^{8}$ Oncology Bioscience, Innovative Medicines and Early Development Biotech Unit, AstraZeneca, Alderley Park, Macclesfield SK10 4TG, UK

${ }^{9}$ European Molecular Biology Laboratory, European Bioinformatics Institute (EMBL-EBI), Wellcome Genome Campus, Hinxton, Cambridgeshire CB10 1SD, UK
\end{abstract}

Acquired resistance to MEK1/2 inhibitors can arise through amplification of $\mathrm{BRAF}^{\mathrm{V} 600 \mathrm{E}}$ or $\mathrm{KRAS} \mathrm{S}^{\mathrm{G} 13 \mathrm{D}}$ to reinstate ERK1/2 signalling. Here we show that $\mathrm{BRAF}^{\mathrm{V} 600 \mathrm{E}}$ amplification and selumetinib resistance are fully reversible following drug withdrawal. Resistant cells with $\mathrm{BRAF}^{\mathrm{V} 600 \mathrm{E}}$ amplification become addicted to selumetinib to maintain a precise level of ERK1/2 signalling (2\%-3\% of total ERK $1 / 2$ active, here quantified by mass spectrometry), that is optimal for cell survival and proliferation. The magnitude of ERK1/2 activation following selumetinib withdrawal ( $20 \%$ active) drives a p $57^{\mathrm{KIP} 2}$-dependent G1 cell cycle arrest and senescence or expression of NOXA and cell death, which selects against those cells with amplified BRAF ${ }^{\mathrm{V} 600 \mathrm{E}}$. ERK1/2-dependent $\mathrm{p}_{5}{ }^{\mathrm{KIP} 2}$ expression is required for loss of BRAF ${ }^{\mathrm{V} 600 \mathrm{E}}$ amplification and determines the rate of reversal of selumetinib resistance. Furthermore, growth of selumetinib-resistant cells with $\mathrm{BRAF}^{\mathrm{V} 600 \mathrm{E}}$ amplification as tumour xenografts is inhibited in mice that do not receive selumetinib. Thus, $\mathrm{BRAF}^{\mathrm{V} 600 \mathrm{E}}$ amplification confers a selective disadvantage during drug withdrawal, providing a rationale for intermittent dosing to forestall resistance. In striking contrast, selumetinib resistance driven by KRAS ${ }^{\mathrm{G} 13 \mathrm{D}}$ amplification is not reversible. In these cells ERK1/2 reactivation does not inhibit proliferation but drives a ZEB1-dependent epithelial-to-mesenchymal transition that increases cell motility and promotes resistance to traditional chemotherapy agents arguing strongly against the use of 'drug holidays' in cases of KRAS ${ }^{\mathrm{G} 13 \mathrm{D}}$ amplification.

\title{
20. Drug repurposing in neuroblastoma to overcome chemoresistance
}

\author{
Madhu Kollareddy ${ }^{1}$, Alice Sherard ${ }^{2}$, Martin Michaelis ${ }^{3}$, Jindrich Cintal $\mathrm{Jr}^{4}$, Abderrahmane Kaidi ${ }^{2}$, \\ Karim Malik ${ }^{1}$
}

${ }^{1}$ Cancer Epigenetics Laboratory, School of Cellular and Molecular Medicine, University of Bristol, Bristol, UK ${ }^{2}$ Nuclear Dynamics Laboratory, School of Cellular and Molecular Medicine, University of Bristol, Bristol, UK ${ }^{3}$ Centre for Molecular Processing and School of Biosciences, University of Kent, Canterbury, UK

${ }^{4}$ Institut für Medizinische Virologie, Klinikum der Goethe-Universität, Frankfurt am Main, Germany 
Despite good initial responses towards non-targeted drugs, $\sim 50 \%$ of children with high-risk neuroblastoma (NB) relapse. Drug-resistance is a major obstacle for successful chemotherapy in high-risk patients. For high-risk subset, new chemotherapeutic agents are urgently required for better response and also to combat resistance. We repurposed an Ewing's sarcoma drug, YK-4-279 (targeted towards EWS-FLI1 oncogenic fusion protein) to target ETS-transcription factors (effectors of ALK and Ras-pathways) which are highly overexpressed in NB. YK-4-279 inhibited growth of wide range of NB genotypes with different oncogenic drivers. Independent of ETS-transcription factors we discovered an unexpected mode of action. YK-4-279 rapidly induced a strong mitotic phenotype. Depending on the cell line, treatment resulted in multipolar, monopolar and fragmented spindles, together leading to disrupted mitotic progression. Notably, YK-4-279 does not affect microtubule acetylation, unlike the conventional mitotic poisons paclitaxel and vincristine. Mechanistically we show that YK-4-279 affected the distribution of Eg5, a key kinesin involved in the fidelity of microtubule assembly. Interestingly YK-4-279 induced robust apoptosis independent of p53 in a caspase-3 dependent manner as confirmed by QVD experiments. With respect to mechanism of toxicity our flow cytometry analysis showed that the majority of cells died in subsequent interphase, not in mitosis. Strikingly YK-4-279 overcame multi-drug resistance in vincristine resistant NB cell lines. Despite extremely high levels of a MDR1/ABCB1 (drug transporter), YK-4-279 was unaffected, suggesting it is not a substrate for MDR1 transporter. We also showed that YK-4-279 effectively synergized with clinical mitotic inhibitors, highlighting the potential of YK-4-279 as an adjuvant chemotherapeutic agent. Thus, YK-4-279 could potentially be used as a single-agent or in combination therapies for the treatment of high-risk and relapsing neuroblastoma, as well as other cancers.

\title{
21. A potential role for IL6ST mediating endocrine resistance in breast cancer via interaction with the ER signaling pathway
}

\author{
Duniya Mosly ${ }^{1,2}$, Arran K. Turnbull, ${ }^{1,2}$, Simon P. Langdon ${ }^{1}$, Andrew H. Sims ${ }^{2}$ \\ ${ }^{1}$ Cancer Research UK Edinburgh Centre and Division of Pathology Laboratory, MRC \\ Institute of Genetics and Molecular Medicine, University of Edinburgh, Edinburgh EH4 2XU, UK \\ ${ }^{2}$ Applied Bioinformatics of Cancer, University of Edinburgh Cancer Research Centre, MRC Institute of Genet- \\ ics and Molecular Medicine, Edinburgh EH4 2XR, UK
}

\begin{abstract}
Aim: IL6ST (gp130) receptor has been identified as a predictive biomarker of endocrine treatment response in breast cancer patients and is included in the "Endopredict" test. At least seven cytokines signal via IL6ST. Interleukin-6 (IL-6) can mediate effects via two signaling pathways; classic signaling (through the membrane-bound IL-6 receptor, IL-6R) and trans-signaling (via non-signaling membrane-bound soluble IL-6R, sIL-6R). Both pathways may occur in parallel and activate cells.
\end{abstract}

Methods: Three ERa+ luminal breast cancer cell lines (MCF-7, T47D, ZR-75-1) were chosen to examine IL6ST expression by western blot, gel electrophoresis and qRT-PCR. Proliferation assays were carried out to investigate the effects of IL- 6 family cytokines on cell growth. The action of both IL-6 and OSM on cell migration and downstream signaling pathways was studied. The extent of trans-signaling occurrence was investigated.

Results: Three cell lines were shown to express varying levels of full length IL6ST. IL6ST soluble forms were identified in the three cell lines. Surprisingly, the growth response to the cytokines was variable across the cell lines. IL6 caused a modest increase in growth in MCF-7 but produced inhibition in ZR-75-1. OSM and LIF stimulated growth in MCF-7, whereas only OSM inhibited ZR-75-1. Interestingly, no significant effect on growth was seen in T47D. Both STAT3 and MAPK/ERK pathways were activated to different 
extents in the three cell lines, whereas cells migration was only stimulated in ZR-75-1. The trans-signaling pathway significantly enhanced the growth inhibition in ZR-75-1.

Conclusion: The presence of both classic and trans-signaling occurrence in breast cancer cell lines. IL6ST full length form is the most abundant form under basal conditions. The levels of the different IL6ST soluble forms will be further studied after estrogen stimulation. The effect of IL6ST silencing in the presence of estrogen and tamoxifen on cell growth and the gene expression is currently being examined.

\title{
22. Computational identification of drug resistance mutations to targeted cancer therapies
}

\section{Teresa Kaserer, Julian Blagg}

Cancer Research UK Cancer Therapeutics Unit, The Institute of Cancer Research, London SM2 5NG, UK

\begin{abstract}
Although targeted cancer therapies have shown great success in the clinic, many patients eventually relapse and develop resistance. The underlying mechanisms are often only dissected in clinical studies, leading to a reactive approach towards drug resistance. We have developed a computational workflow combining drug affinity, clonal fitness and mutational signatures to prioritize resistance mutations in the primary drug targets, which are likely to arise upon treatment of a specific cancer type. This workflow has been validated on a comprehensive ERK2 benchmark literature dataset and applied to successfully identify clinicallyobserved resistance mutations for approved drugs targeting Kit, Abl, EGFR, and ALK. Our results suggest that this workflow can be prospectively applied to signpost likely resistance mutations and thereby support improved patient monitoring and the timely development of effective next-generation drugs to manage resistance to targeted therapies.
\end{abstract}

\section{Loss of BAP1 diminishes sensitivity to vinorelbine in Mesothelioma}

\author{
Anita Singh, Dean A. Fennell, Andrew M. Fry \\ University of Leicester, University Road, Leicester LE1 7RH, UK
}

\begin{abstract}
Aim: BRCA1 associated protein 1 (BAP1) is a tumor suppressor that is commonly inactivated in the majority of mesotheliomas. We have previously reported that BRCA1 is lost in $38 \%$ of mesothelioma cases and leads to vinorelbine resistance. However the mechanism of BRCA1 loss is unknown. Vinorelbine, a spindle checkpoint activator that perturbs microtubule dynamics has shown clinical efficacy with a response rate of $24 \%$, whereas no other chemotherapeutic agents used in treatment of mesothelioma had a response rate greater than $20 \%$. The aim of the study was to explore the functional relationship between BAP1 and BRCA1 and their role in vinorelbine resistance.
\end{abstract}

Methods: We conducted functional analysis of BAP1 and BRCA1 in MSTO (wild-type BAP1), H2452 (A95D mutation in the UCH domain), and $\mathrm{H} 226$ ( BAP1 null cell line) along with expression vectors carrying wild-type or mutant forms of BAP1. BAP1 knockdown was achieved by siRNA transfection in MSTO and $\mathrm{H} 2452$, while BRCA1 knockdown was achieved by doxycycline induction of an integrated shRNA in MSTO.

Results: Loss of BAP1 expression led to reduced expression of BRCA1, whereas knockdown of BRCA1 did not affect BAP1 expression. Treatment with the proteasome inhibitor, MG132, restored BRCA1 expression in the absence of BAP1 indicating that BAP1 contributes to post-translational stabilization of BRCA1 protein. Loss of BAP1 resulted in vinorelbine resistant, a phenotype similar BRCA1 deficiency. BAP1 also promotes BRCA1 foci formation after DNA damage. 
Conclusion: Our data demonstrate that BAP1 controls BRCA1 expression through regulating its protein stability. Bap1 status is a major determinant of BRCA1 expression and in efficacy microtubule poisons such as vinorelbine, a hypothesis that we will test in our randomised Medusa and VIM trial.

\section{Investigating resistance mechanisms to CB-839 in RCC using a genome-wide CRISPR/ Cas9 approach}

\section{Aleksandra Raczka}

Medical and Biological Sciences Building, University of St Andrews, North Haugh, St Andrews, Fife KY16 $9 T F, U K$

Renal cell carcinoma (RCC) affects 1 in 82 men and 1 in 57 women, and accounts for about 3\% of all cancer deaths worldwide. It is characterised by significant tumour heterogeneity and inherent (in 25\%-30\% cases) or acquired resistance to available chemotherapeutics. Most patients who initially respond to treatment develop resistance within 6-15 months. Glutamine addiction is a potential new therapeutic target for kidney cancers. These cancer cells use glutamine rather than glucose to meet their enhanced bioenergetics demands to sustain their rapid growth. They are very often deficient in functional von Hippel Lindau (VHL) protein, which leads to abnormal accumulation of several proteins involved in angiogenesis and cell division. VHL-/- tumours have been recently shown not only to rely on glutamine for energy generation and maintenance of redox homeostasis, but also for de novo pyrimidine synthesis. CB-839 is a small, orally administered reversible inhibitor of human kidney-type glutaminase (GLS). GLS converts glutamate to glutamine and is upregulated in glutamine-addicted cancers, such as RCC and triple negative breast cancer (TNBC). CB-839 is currently in clinical trials as a monotherapy and also as part of a combination therapy, and shows promising results. Given the significant incidence of resistance to previously approved therapies, we are applying a genome-wide CRISPR/Cas9 approach to identify candidate genes, which when knocked down will confer resistance to CB-839. We are screening the genome-scale GeCKOv2 sgRNA library, previously used to identify genes involved in resistance to vemurafenib in melanoma cells. Since most RCCs are VHL-/-, we are using 786-O cells as a model of RCC where CB-839 inhibits their growth. This screen should identify candidate genes mediating resistance to $\mathrm{CB}-839$ that could be useful as biomarkers or as part of rational drug combinations.

\section{Multivariate variable selection for integrative analysis of cancer drug data}

\section{Jian Zhang, Elaheh Oftadeh}

\section{School of Mathematics, Statistics and Actuarial Science, University of Kent, Canterbury CT2 7FS, UK}

Cancer drugs exert their function through binding to one or more protein targets (Wang et al., 2014). Early "one gene, one drug, one cancer" paradigm considers the role of individual genes and their changes in drug-perturbed states, which largely ignore a target's cellular and physiological context. Meanwhile, cancer gene-centric methods largely ignore the multi-factor-driven attribute of cancer diseases at the cellular level. With the generation of rich data resources for genome-wide gene expressions and drug- and cancerinduced perturbations, we propose an integrative analysis of these data to provide systematic insights into mechanisms of drugs and cancers in a "multiple genes, multiple drugs, multiple types of cancers" paradigm. The proposed method, called principal variable analysis, aims at selecting predictor variables with relatively higher coefficient variations in a multivariate regression model. The basic premise behind the proposal is to scan through a predictor variable space with a series of forward filters named null- 
beamformers; each is tailored to a particular region in the space and resistant to interference effects originating from other regions. The new approach attempts to explore the maximum amount of variation in the data with a small number of principal variables. Through simulation studies, we show that it substantially outperforms the existing methods in terms of sensitivity and specificity. We apply the proposed method to $\mathrm{IC}_{50}$ cancer drug and gene expression datasets, identifying a set of important genes co-associated with a group of cancer drugs.

\title{
26. Targeting of a transcriptional state implicated in resistance to MEK inhibition in KRAS- mutant colorectal cancer
}

Steve Wagner ${ }^{1}$, Georgios Vlachogiannis ${ }^{2}$, Alexis De Haven Brandon ${ }^{1}$, Melanie Valenti ${ }^{1}$, Gary Box $^{1}$, Liam Jenkins ${ }^{3}$, Caterina Mancusi ${ }^{1}$, Annette Self ${ }^{1}$, Floriana Manodoro ${ }^{4}$, loannis Assiotis ${ }^{4}$, Penny Robinson ${ }^{4}$, Ritika Chauhan ${ }^{4}$, Alistair G Rust ${ }^{4}$, Nik Matthews ${ }^{4}$, Kate Eason ${ }^{2}$, Khurum Khan $^{5}$, Naureen Starling ${ }^{5}$, David Cunningham ${ }^{5}$, Anguraj Sadanandam ${ }^{2}$, Clare M. Isacke ${ }^{3}$, Vladimir Kirkin $^{1}$, Nicola Valeri ${ }^{2,5}$, Steven R. Whittaker ${ }^{1}$

\author{
${ }^{1}$ Division of Cancer Therapeutics, The Institute of Cancer Research, London, UK \\ ${ }^{2}$ Division of Molecular Pathology, The Institute of Cancer Research, London, UK \\ ${ }^{3}$ Breast Cancer Now Research Centre, The Institute of Cancer Research, London, UK \\ ${ }^{4}$ Tumour Profiling Unit, The Institute of Cancer Research, London, UK \\ ${ }^{5}$ Department of Medicine, Royal Marsden NHS Foundation Trust, London, UK
}

The MEK inhibitor (MEKi) trametinib has shown clinical benefit in BRAF-mutant melanoma but showed no clinical responses in KRAS-mutant colorectal cancer. To gain insight into potential mechanisms of resistance, we used a pharmacogenomics analysis of colorectal cancer cell lines exhibiting differential sensitivity to MEK inhibition. Strikingly, we identified a transcriptional state, involving increased interferon and inflammatory gene expression, to be highly enriched in cell lines displaying intrinsic and acquired resistance to MEK inhibition. Inhibition of this transcriptional state was achieved by small molecule inhibition of the bromodomain family of proteins, which suppressed interferon and inflammatory gene expression and restored sensitivity to MEK inhibition. The combination of the bromodomain inhibitor JQ1 and trametinib showed synergistic anti-proliferative effects and induced apoptosis in MEKi-resistant colorectal cancer cell lines. Elevated expression of interferon/inflammatory genes was observed in patient-derived organoid models which were also resistant to trametinib and were resensitized by co-treatment with JQ1. In both xenograft and syngeneic models of colorectal cancer, the combination of trametinib and JQ1 significantly suppressed tumour growth compared to either agent alone. Furthermore, using a 66-gene signature we found that high expression of interferon/inflammatory genes was associated with significantly reduced survival of colorectal cancer patients. These findings provide a novel explanation for the lack of response to MEK inhibitors in KRAS-mutant colorectal cancer, known for its inflammatory nature. Importantly, we have identified a novel therapeutic strategy to overcome both intrinsic and acquired resistance to MEK inhibition in colorectal cancer.

27. A genome-wide CRISPR screen to identify combination strategies for AZD5353 (AKTi) and AZD8186 (PI3K $\beta \mathrm{i})$ in PTEN-null breast cancer

Shanade Dunn ${ }^{1,4}$, Jason $\mathrm{Yu}^{4}$, Swee Hoe Ong ${ }^{4}$, Yan Zi Au ${ }^{4}$, Urs Yelland ${ }^{2}$, Natalie Cureton ${ }^{2}$, Anna Staniszewsla ${ }^{1}$, James Pilling ${ }^{3}$, Philip Hopcroft ${ }^{3}$, Beverley Isherwood ${ }^{3}$, Simon Barry ${ }^{1}$, Barry Davies $^{1}$, James Lynch ${ }^{1}$, Kosuke Yusa ${ }^{4}$ 
${ }^{1}$ Bioscience, Oncology, IMED Biotech Unit, AstraZeneca, Cambridge, UK

${ }^{2}$ Bioscience, Oncology, IMED Biotech Unit, AstraZeneca, Alderley Park, UK

${ }^{3}$ Discovery Sciences, IMED Biotech Unit, AstraZeneca, Cambridge, UK

${ }^{4}$ Wellcome Trust Sanger Institute, Cambridge, UK

The PI3K-AKT-mTOR pathway is frequently hyper-activated in breast cancer and several inhibitors targeting the PI3K pathway, including AKT (AZD5363) and PI3K $\beta$ (AZD8186), are in clinical development. A better understanding of the mechanisms of resistance to PI3K pathway inhibitors is critical for the development of combination strategies. To this end, we performed genome-wide CRISPR-Cas9 knockout screens to identify genes that mediate drug resistance to AZD5363 and AZD8186 in three PTEN-null breast cancer cell lines. The screens have confirmed a number of known resistance genes and also revealed a number of novel mediators. Moreover, our screens identified a number of "sensitiser" genes. Eighteen of these hits have inhibitors in clinical development and we have performed a combination screen to identify the compounds that give the best synergy with AZD5363/AZD8186 across the breast cancer cell lines. The most striking combination was with the Mcl-1 inhibitor, AZD5991. Mechanistic work is ongoing and our data suggest that AZD5363/AZD8186 "prime" cells for apoptosis and combined inhibition with AZD5991 drives a rapid apoptotic response. Furthermore, these combinations induce regressions in a breast cancer xenograft model. Overall, our CRISPR screening data provide new insights into the pathways that drive resistance and have identified novel synergistic combinations.

\title{
28. Acquired resistance mechanisms to $\beta$-tubulin inhibitors in triple negative breast cancer
}

\author{
Helen E. Grimsley ${ }^{1}$, Jindrich Cinatl $\mathrm{Jr}^{2}$, Catherine Harper-Wynne ${ }^{3}$, Karina Cox ${ }^{3}$, Martin \\ Michaelis ${ }^{1}$, Mark N. Wass ${ }^{1}$, Michelle D. Garrett ${ }^{1}$ \\ ${ }^{1}$ School of Biosciences, University of Kent, Canterbury, UK \\ ${ }^{2}$ Institut für Medizinische Virologie, Klinikum der Goethe-Universität, Frankfurt am Main, Germany \\ ${ }^{3}$ Maidstone and Tunbridge Wells NHS Trust
}

Microtubules are dynamic components of the cytoskeleton, and are common targets for anticancer therapies. Disruption of mitotic processes through inhibition of $\beta$-tubulin, an essential building block of the mitotic spindle, leads to mitotic catastrophe. $\beta$-tubulin inhibitors; Eribulin and Paclitaxel are common drugs for the treatment of triple negative breast cancer. Triple negative breast cancer exhibits an aggressive metastatic phenotype and patients are commonly known to relapse due to acquired drug resistance. Here we start to elucidate mechanisms of resistance to $\beta$-tubulin inhibitors which may provide opportunities to strategically alter their therapeutic use in patients. In vitro assays demonstrated similar phenotypes in the Eribulin-adapted and Paclitaxel-adapted cell lines, distinct from the parental. Cross-resistance was found upon treatment with Eribulin and Paclitaxel in the drug-adapted cell lines. Remarkably, whole exome sequencing analysis showed only a small number of variants gained in the drug-adapted cell lines, with even less shared in respective drug-adapted cell lines. Our results suggest that although similar responses in the drug-adapted cell lines are seen when treated with Eribulin and Paclitaxel, the acquired resistance mechanisms are most likely to be different.

\section{The role of SOX4 in drug-resistant chronic myeloid leukemia}

\section{Jorge Luis Jimenez Macias, April J. Baral, Peter Laslo}

Section of Experimental Haematology, Leeds Institute of Molecular Medicine, St James's University Hospital, Leeds, UK 
Chronic myeloid leukaemia (CML) is associated with the BCR-ABL1 (BA) oncogene and managed with drug therapy [Imatinib (IM)]. However, $20 \%$ of patients will develop drug resistance resulting in fatality within 12 months. Previous work in the lab had established a preclinical model of CML drug resistance using KCL22 cells. By gradually exposing these cells to increasing concentrations of IM, a drug-resistant derivative (IMr) was established. The IMr cells recapitulated clinical observations where drug treatment ablated the activity of BA yet they continue to grow. In order to survive the IMr cells must acquire new oncogenic drivers that compensate for the loss of BA. Research focused on the functional requirement of the SOX4 factor in establishing the IMr phenotype. Depletion of SOX4 within the IMr cells inhibited cell growth. Interestingly, the complimentary gain-of-function studies failed to generate SOX4-expressing KCL22 stables. Targeting SOX4 with the aim of restoring its normal functional activity holds great promise for the therapeutic treatment of drug-resistant CML.

\title{
30. PHLDA1 downregulation mediates drug resistance in receptor tyrosine kinase-driven cancer and can be modulated using HDAC Inhibitors
}

\author{
N. S. Clayton ${ }^{1}$, E. P. Carter ${ }^{1}$, A. E. Fearon ${ }^{2}$, J. A. Heward ${ }^{3}$, R. P. Grose ${ }^{1}$ \\ ${ }^{1}$ Centre for Tumour Biology, Barts Cancer Institute - a CRUK Centre of Excellence, Queen Mary University of \\ London, London EC1M 6BQ, London, UK \\ ${ }^{2}$ Inst. f. Molecular Health Sciences, Department of Biology, ETH Zürich, Zürich 8093, Switzerland \\ ${ }^{3}$ Centre for Haemato-Oncology, Barts Cancer Institute, London EC1M 6BQ, UK
}

Development of resistance causes failure of drugs targeting oncogenic receptor tyrosine kinases (RTKs) and represents a critical challenge for precision medicine. Here, we show that transcriptional downregulation of the PH domain-containing protein PHLDA1 is critical to the acquisition and maintenance of drug resistance in RTK-driven cancer. Using resistance to fibroblast growth factor receptor - targeted therapy as a model, we identified an Akt-driven compensatory mechanism underpinned by downregulation of PHLDA1. We demonstrate broad clinical relevance of our findings, showing that PHLDA1 downregulation also occurs in response to RTK-targeted therapy in breast and renal cancer patients. Crucially, knockdown of PHLDA1 alone was sufficient to confer de novo resistance to RTK inhibitors in vitro and induction of PHLDA1 expression re-sensitized drug-resistant cancer cells to targeted therapies. This identifies PHLDA1 as a biomarker for drug response and highlights the potential of PHLDA1 reactivation as a means of circumventing resistance to RTK inhibitors. We have identified inhibition of the MAPK pathway as a crucial step in PHLDA1 downregulation and show that ERK1/2 inhibition produces significant epigenetic changes at the PHLDA1 locus, specifically, a decrease in the activatory marks $\mathrm{H} 3 \mathrm{Kme} 3$ and $\mathrm{H} 3 \mathrm{~K} 27 \mathrm{ac}$. In line with this, we show that treatment with clinically relevant Class I HDAC inhibitors restores PHLDA1 expression in resistant cells. Using HER $2^{+}$breast cancer cells as a model system, we have shown that HDAC inhibitors increase the sensitivity of resistant cells to HER $2^{+}$-targeted therapy and when given in combination with lapatinib, produce a synergistic effect on cell growth in parental cell populations.

\section{Elucidating mechanisms of resistance to PI3K inhibition in pancreatic cancer using functional genomics and combinatorial drug screening}

\section{Charlotte Milton ${ }^{1}$, Annette Self ${ }^{1}$, Rosemary Burke ${ }^{1}$, Federica Piccioni ${ }^{2}$, David Root ${ }^{2}$, Steven R. Whittaker ${ }^{1}$}

\author{
${ }^{1}$ Division of Cancer Therapeutics, The Institute of Cancer Research, London, UK \\ ${ }^{2}$ Genetic Perturbation Platform, The Broad Institute, Cambridge, MA, USA
}

Activating mutations in the oncogene KRAS are present in $95 \%$ of pancreatic ductal adenocarcinoma 
(PDAC) and drive tumorigenesis and disease progression. The PI3K pathway is activated downstream of oncogenic KRAS signalling and presents an attractive therapeutic target for the treatment of this lethal disease. However, when tested in PDAC clinical trials, PIзK pathway inhibitors have thus far derived no significant benefit. We hypothesise that an adaptive response mediates resistance to PI3K inhibition in PDAC, targeting of which may present a novel therapeutic strategy. We found that resistance to the PI3K alpha inhibitor, BYL719, is associated with sustained PI3K-independent activation of mTORC1 in a panel of PDAC cell lines. We demonstrate that inhibition of mTOR, in combination with PI3K inhibition, is necessary to fully supress PI3K signalling and inhibit proliferation of PDAC cells. Through genome-scale and focussed, mini-pool CRISPR screens, we nominated key genes in the mTOR and RTK signalling networks responsible for mediating resistance and show that siRNA mediated knockdown of these genes is sufficient to sensitise to PI3K alpha inhibition in multiple cell lines. Notably, over-expression of multiple genes involved in determining PI3K inhibitor sensitivity is associated with significantly decreased patient survival. Targeting of multiple nodes in the PI3K signalling network is therefore essential to overcoming resistance to single agent $\mathrm{PI} 3 \mathrm{~K}$ alpha inhibition. In 3D stromal co-culture models, inhibition of either mTOR, EGFR or PDK1 synergistically inhibited spheroid growth in combination with PI3K alpha inhibition. Our work therefore supports further investigation into combined PI3K and RTK inhibition for the treatment of pancreatic cancer.

\title{
32. A predictive model of "on-target" drug resistance (for poster)
}

\section{Daniel O'NeilI ${ }^{1,3}$, Maryam Clausen ${ }^{2}$, Mike Firth $^{1}$, Jonathan Wrigley ${ }^{1}$, Maria Emanuela Cuomo ${ }^{1}$}

\author{
${ }^{1}$ AstraZeneca, IMED Discovery Sciences, Cambridge, UK \\ ${ }^{2}$ AstraZeneca, IMED Discovery Sciences, Gothenburg, SE \\ ${ }^{3}$ AstraZeneca, IMED Oncology, Cambridge, UK
}

Acquired drug resistance represents a major challenge in the development of therapeutic agents for the treatment of cancer. The problem is exemplified by non-small cell lung cancer (NSCLC) containing activating EGFR mutations. NSCLC tumours driven by EGFR variants are initially sensitive to tyrosine kinase inhibitors (TKI), but some patients show renewed disease progression through expansion of tumour cell clones harbouring additional EGFR mutations. Models of drug resistance are therefore necessary to increase our understanding of resistance mechanisms, predict variants that will arise in patients and improve drug development strategies. In the past, this problem has been addressed by screening large panels of gene variants for drug resistance phenotypes in cell culture. Previous methods such as chemical mutagenesis has the advantage of testing mutations genome wide but did not cover all possible amino acid variants in a specific region of interest. On the other hand, saturation mutagenesis with "on target" cDNA libraries resulted in expression of all possible variants but in a non-physiological setting, detached from their endogenous regulatory mechanisms. For the first time, CRISPR/Cas9 allows endogenous modification of genes and facilitates introduction of all possible mutations at a specific locus, whilst maintaining endogenous gene architecture, chromosomal context and epigenetic mileu. Here we describe the novel CRISPRbased methodology targeted endogenous mapping of pharmacological resistance developed to identify "on target" mutations in key oncogenes that drive pharmacological resistance. Using EGFR as a target, we show how directed evolution of some residues in the EGFR kinase domain can be predictive of the resistance observed in the clinic in response to known TKIs, such as gefitinib and osimertinib. In fact, in the region analysed, we can recapitulate the variants observed clinically. We also identify amino acids chemically and structurally able to confer resistance to a TKI in structurally conserved tyrosine kinases such as BCR-Abl and Kit. 
33. A FGF7-FGFR2 autocrine loop maintains fusion positive rhabdomyosarcoma cell growth and is associated with preclinical efficacy of the FGFR inhibitor NVP-BGJ398

Christopher I. Milton ${ }^{1}$, Joanna Selfe ${ }^{1}$, Edoardo Missiaglia ${ }^{2}$, Ewa Aladowicz ${ }^{1}$, Zoë S. Walters ${ }^{1}$, Susanne A. Gatz ${ }^{1}$, Melanie Generali ${ }^{6}$, Gary Box ${ }^{3}$, Melanie Valenti ${ }^{3}$, Alexis de Haven-Brandon ${ }^{3}$, David Galiwango ${ }^{4}$, Angela Hayes ${ }^{4}$, Matthew Clarke ${ }^{5}$, Elisa Izquierdo ${ }^{5}$, David Gonzalez De Castro ${ }^{6}$, Florence Raynaud $^{4}$, Suzanne Eccles ${ }^{3}$, Janet M. Shipley ${ }^{1}$

${ }^{1}$ Sarcoma Molecular Pathology Team, Divisions of Molecular Pathology and Cancer Therapeutics, The Institute of Cancer Research, London, UK

${ }^{2}$ Department of Molecular Pathology, Centre Hospitalier Universitaire Vaudois, Lausanne CH-1011, Switzerland

${ }^{3}$ Tumour Biology and Metastasis Team, Division of Cancer Therapeutics, The Institute of Cancer Research, London, UK

${ }^{4}$ Drug Metabolism and Pharmacokinetics Team, Division of Cancer Therapeutics, The Institute of Cancer Research, London, UK

${ }^{5}$ Glioma Team, Division of Molecular Pathology, The Institute of Cancer Research, London, UK

${ }^{6}$ Molecular Haematology, Division of Molecular Pathology, The Institute of Cancer Research, London, UK; current address for Melanie Generali: Center for Therapy Development (GMP), Institute for Regenerative Medicine (IREM), University of Zurich, Zurich CH-8044, Switzerland; and current address for David Gonzalez De Castro: School of Medicine, Dentistry and Biomedical sciences, Queens University Belfast, Belfast BT9 $7 B L, U K$

Rhabdomyosarcoma (RMS) is the most common form of soft tissue sarcoma in children. Patients whose tumours harbour a genetic fusion, the most common of which is PAX3-FOXO1, carry the worst prognosis with a 5-year survival rate of less than $30 \%$. Evidence demonstrates that fibroblast growth factor receptor 4 (FGFR4) plays a role in fusion positive RMS cell growth, although it is not clear whether other family members (FGFRs 1-3) contribute as well. We sought to validate FGFR involvement in RMS proliferation and to provide preclinical evidence that inhibiting FGFR signalling is a viable therapeutic strategy in this tumour type. From a RMS cell line screen we identified fusion positive cells as more sensitive than fusion negative cells to the FGFR inhibitor and clinical candidate NVP-BGJ398. Sensitivity correlated with high mRNA expression of FGFR2 and its specific ligand FGF7. Genetic knockdown of FGF7 and FGFR2 decreased fusion positive but not fusion negative RMS cell viability. Interestingly, levels of secreted FGF7 protein were maintained alongside FGFR signalling through the MAPK pathway upon serum starvation. This indicates that fusion positive RMS growth in vitro is sustained by a FGF7-FGFR2 autocrine loop. We mined expression data from three independent RMS patient cohorts which revealed overexpression of FGFR2 and FGF7 in fusion positive tumours compared to fusion negative tumours and normal muscle. Oral administration of NVP-BGJ398 to immunodeficient mice, at doses within the range achievable in humans, significantly inhibited fusion positive RMS01 and RH41 xenograft tumour growth, concomitant with diminished FGFR/MAPK signalling and a lower proliferative index. Finally, using clonogenic assays in vitro we demonstrate that NVP-BGJ398 is synergistic in combination with Irinotecan, a chemotherapeutic used in the standard treatment of RMS patients. Together these data suggest that NVP-BGJ398 is an attractive therapeutic option, particularly in combination with Irinotecan, for high-risk fusion positive RMS patients.

\section{Exploring resistance of renal cell carcinoma to targeted therapy}

\section{Hossam M. Kamli ${ }^{1,2}$, Li Li ${ }^{3}$, David A. Vesey ${ }^{1,4}$, Glenda C. Gobe ${ }^{1,5}$}

${ }^{1}$ University of Queensland Princess Alexandra Hospital Kidney Disease Research Collaborative, Translational Research Institute, Brisbane, Queensland, Australia 
${ }^{2}$ Department of Clinical Laboratory Sciences, College of Applied Medical Sciences, King Khalid University, Abha, Saudi Arabia

${ }^{3}$ UQ-Ochsner Clinical School, Ochsner Clinic Foundation, New Orleans, LA, USA

${ }^{4}$ Departments of Urology and Nephrology, Princess Alexandra Hospital, Woolloongabba, Brisbane, Australia

${ }^{5} U Q$ NHMRC CKD.QLD CRE, Royal Brisbane and Women's Hospital, Brisbane, Australia

Renal cell carcinoma (RCC) accounts for 2\%-3\% of all adult malignancies and its incidence has risen over past decades. If untreated, RCC is associated with high rates of metastasis and high mortality, partially due to high levels of drug resistance. The novel gold-standard tyrosine kinase inhibitor, Sunitinib, targets angiogenesis and other multiple pathways in RCC treatment. Sunitinib-resistance is a major clinical problem for treatment of RCC. The comprehensive characterisation of morphological, functional and molecular changes in Sunitinib-resistant RCC cells is lacking. We aimed to develop Sunitinib-resistant human RCC cell lines (SN12K1, Caki-1, Caki-2 and 786-0) and characterise the cellular biological changes. RCC cells were made resistant by continuous, chronic exposure to $10 \mu \mathrm{mol} / \mathrm{L}$ Sunitinib. Resistant RCC cell lines were stable in $10 \mu \mathrm{mol} / \mathrm{L}$ Sunitinib for nearly 2 years, while in parental cell lines, $10 \mu \mathrm{mol} / \mathrm{L}$ Sunitinib induced 95\%-98\% cell death at 72-h. Cell proliferation, morphology, transmigration, a cellular adhesion assay, and gene expression (Q-RT-PCR) for vascular endothelial growth factor (VEGF), interleukin (IL)-6, IL-8, Bcl2 and Bax were studied. There was no significant difference in growth rate and transmigration between the parental and resistant cells. Sunitinib-resistant cells were significantly hypertrophic compared with parental cells as evidenced by increases in the surface areas of the whole cells and the nuclei. VEGF was increased in Caki-2 and SN12K1 resistant cells by 5 and 2 -fold, respectively. IL- 6 was increased significantly in 786-0, Caki-1, Caki-2 and SN12K1 resistant cells by 3.5, 2, 2 and 12-fold, respectively. IL-8 increased in Sunitinib-resistant Caki-2 and $\mathrm{SN} 12 \mathrm{~K} 1$ cells by 2 and 2.5 -fold, respectively. The Bcl2/Bax ratio increased in Caki-1, Caki-2, and SN12K1 cells by 1.5, 2, and 2-fold, respectively, indicating increased apoptosis resistance. Elevated IL-6 may contribute to Sunitinib resistance either via VEGF-mediated angiogenesis or antiapoptotic Bcl2. Thus inhibition of IL- 6 could be a promising way to overcome drug resistance.

\title{
35. PEA-15 unphosphorylated at both serine 104 and serine 116 sensitizes ovarian carcinoma cells to cisplatin
}

\section{Shahana Dilruba', Anke C. Schiedel ${ }^{2}$, Naoto T. Ueno ${ }^{3}$, Florian Rothweiler ${ }^{4}$, Jindrich Cinatl $\mathrm{Jr}^{4}$, Martin Michaelis ${ }^{5}$, Ganna V. Kalayda ${ }^{1}$}

\author{
${ }^{1}$ Institute of Pharmacy, Clinical Pharmacy, University of Bonn, Germany \\ ${ }^{2}$ Institute of Pharmacy, Pharmaceutical Chemistry I, University of Bonn, Germany \\ ${ }^{3}$ Department of Breast Medical Oncology, The University of Texas MD Anderson Cancer Center, Houston, \\ TX, USA \\ ${ }^{4}$ Institute of Medical Virology, Goethe University Hospital Frankfurt, Frankfurt/Main, Germany \\ ${ }^{5}$ Industrial Biotechnology Centre and School of Biosciences, School of Biosciences, University of Kent, Canter- \\ bury, UK
}

Cisplatin belongs to the standard chemotherapy regimens of various solid tumors. extracellular signalregulated kinase 1 and 2 (ERK1/2) is activated following cisplatin treatment. Upon activation, ERK1/2 shuttles between different cellular compartments, where it acts on various targets. Intracellular trafficking of ERK1/2 is regulated, among others, by a small scaffold protein PEA-15 (phosphoprotein enriched in astrocytes-15kDa). Depending on its phosphorylation at serine 104 and serine 116, PEA-15 can sequester ERK1/2 in the cytoplasm or facilitate its nuclear translocation. In addition, PEA-15 mediates other cellular processes such as autophagy and apoptosis. These functions of PEA-15 have also controlled its phosphory- 
lation status. Here we have investigated the hypothesis that, depending on the phosphorylation state, PEA15 can enhance cisplatin sensitivity in ovarian cancer cells. Two mutated versions of PEA-15 mimicking the two phosphorylation statuses of the protein were overexpressed in SKOV-3 human ovarian carcinoma cells by Lipofectamine-mediated transfection. PEA-15AA is a mutated non-phosphorylatable form of PEA15 capable of ERK1/2 binding in the cytoplasm, whereas PEA-15DD is a phosphomimetic variant stimulating ERK1/2 nuclear translocation. PEA-15AA overexpression resulted in a significant increase $(P<0.0001)$ in cisplatin sensitivity $\left(\mathrm{EC}_{50}=10.0 \mu \mathrm{mol} / \mathrm{L}\right)$ compared to the mock-transfected cells $\left(\mathrm{EC}_{50}=14.9 \mu \mathrm{mol} / \mathrm{L}\right)$ as determined by an MTT-assay. In PEA-15DD-transfected cells, cisplatin cytotoxicity $\left(\mathrm{EC}_{50}=14.3 \mu \mathrm{mol} / \mathrm{L}\right)$ was similar to that of the mock-transfected controls. The whole-transcriptome expression profiling with a Clariom $^{\mathrm{TM}} \mathrm{S}$ microarray revealed the Nrf2/ARE signaling pathway as central to the PEA-15AA positive effect on the sensitivity of SKOV-3 cells to cisplatin. The relevance of this pathway was successfully validated using retinoic acid, which is a potent inhibitor of Nrf2/ARE. Co-treatment with $20 \mu \mathrm{mol} / \mathrm{L}$ retinoic acid significantly $(P<0.0001)$ sensitized SKOV-3 cells to cisplatin as $\mathrm{EC}_{50}$ was reduced from $32.6 \mu \mathrm{mol} / \mathrm{L}$ for the platinum complex alone to $14.0 \mu \mathrm{mol} / \mathrm{L}$ in the combination. Our results show that non-phosphorylatable PEA-15 increases cisplatin sensitivity in SKOV-3 human ovarian carcinoma cells through interference with the Nrf2/ARE signaling.

\title{
36. Identifying a potential role for TauT in conferring resistance to standard treatment in glio
}

\section{blastoma}

\section{Nora Rippaus ${ }^{1}$, Julie Higgins ${ }^{1}$, Rhiannon Barrow ${ }^{1}$, Alexander Bruns ${ }^{1}$, Aruna Chakrabarty ${ }^{2}$, Azzam Ismail $^{2}$, Jacquelyn Bond ${ }^{1}$, Katherine Ashton ${ }^{3}$, Khaja Syed ${ }^{4}$, Michael Jenkinson ${ }^{4,5}$, Andrew Brodbelt $^{4}$, Susan Short ${ }^{1}$, Lucy F. Stead ${ }^{1}$}

\author{
${ }^{1}$ Faculty of Medicine and Health, St James's University Hospital, University of Leeds, Leeds LS9 7TF, UK \\ ${ }^{2}$ Leeds Teaching Hospitals NHS Trust, St James's University Hospital, Leeds LS9 7TF, UK \\ ${ }^{3}$ Lancashire Teaching Hospitals NHS Trust, Royal Preston Hospital, Preston PR2 9HT, UK \\ ${ }^{4}$ Walton Centre NHS Trust, Liverpool L9 7LJ, UK \\ ${ }^{5}$ Institute of Translational Medicine, University of Liverpool, Liverpool L9 7LJ, UK
}

Glioblastoma (GBM) is an incurable and fatal brain cancer; almost half of patients die within a year of diagnosis despite a standard, aggressive treatment regimen of surgery, radiotherapy and chemotherapy with Temozolomide. All drugs designed to target molecular features of primary GBM tumours have failed to prolong survival. This is likely because of inherently treatment-resistant cells within primary tumours that evade current therapies, continuing to proliferate to form a recurrent tumour. We have sequenced RNA from paired pre- and post-treatment GBM patient tumours to identify genes that are differentially expressed between primary and recurrent GBMs and investigate whether treatment resistance is driven at the transcriptional rather than genomic level, as suggested from recent published work. In parallel, we isolated cancer cells from a newly resected primary GBM and cultured them as polyclonal spheroids in stem-cell permissive conditions. Half of these spheroids received the non-surgical components of standard treatment and seven days later we sequenced RNA from single cells from both treated and untreated spheroids. SLC6A6 was the most significantly upregulated gene in recurrent $v s$. matched primary patient tumours and the only gene that was also significantly upregulated in treated $v s$. untreated single cells. One interpretation of these data is that GBM cells expressing SLC6A6 are more resistant to standard treatment. This is supported by: (1) the fact that patients with primary GBM tumours with low SLC6A6 expression have significantly better prognosis; (2) work showing that SLC6A6 expression promotes multidrug resistance in colorectal cancer; (3) preliminary data from functional screens that we have performed on established GBM cell lines. SLC6A6 encodes a taurine transporter, TauT, with an important role in brain development 
for which we postulate a mechanism for conferring treatment resistance in GBM, the testing of which is the focus of our ongoing work.

\section{Single-cell analysis of cancer-associated fibroblast heterogeneity in non-small cell lung cancer: mapping molecular phenotypes in tumours}

\section{Sara Waise, Rachel Parker, Matthew Rose-Zerilli, Christian Ottemsmeier, Christopher Hanley\#, Gareth Thomas"}

Cancer Sciences Unit, Somers Building, University of Southampton, Tremona Road, Southampton SO16 6YD, UK

"The two authors contributed equally to this Abstract

Aim: To characterise the heterogeneity and spatial relationships of the cancer-associated fibroblast (CAF) population in non-small cell lung cancer.

Methods: Fresh primary lung tissue was dissociated to extract the maximum possible proportion of CAF (using collagenase P for sixty minutes). Single-cell RNA sequencing was performed using a droplet-barcoded sequencing platform. Bioinformatic analysis was carried out in $\mathrm{R}$, with cell type identification and gene set enrichment using the ToppFun tool and GSEA program. Spatial relationships between cell types were characterised using a multi-immunohistochemical staining protocol.

Results: Analysis of 12 non-small cell lung cancer (NSCLC) tumours and 6 matched non-involved lung samples revealed the presence of 5 CAF subtypes. Three subgroups showed transcriptomic overlap with normal fibroblasts. Of the distinct CAF populations, one showed enrichment and expression of genes associated with the 'myofibroblastic' CAF phenotype. The second CAF cluster showed higher expression of growth factors and immune cell exclusion on histology. Multiplexed IHC using identified cluster markers demonstrated that these subtypes have different spatial distribution and relationships to other cell types. Of the remaining three subtypes, one showed gene expression and enrichment consistent with the previously-described 'inflammatory' fibroblast subtype. The remaining two clusters show significant prognostic impact, but their functions have yet to be elucidated.

Conclusion: Despite their abundance in most solid cancers, CAF remain a poorly-characterised population. No single marker reliably identifies all CAF and is not yet clear whether distinct CAF phenotypes exist or whether such subgroups have different functions. We have identified six stromal populations in NSCLC. The fibroblast subtypes show differential gene set enrichment, spatial distribution and prognostic impact, suggestive of functional differences. Clinical trials of stromal targeting agents have so far shown disappointing results: identification of pro-malignant CAF subgroups may facilitate the development of more specific strategies.

\section{The unfolded protein response in prostate cancer: developing novel strategies for therapeutic intervention}

\section{Ana Maria Isac, Filippo Prischi, Philip Reeves, Greg Brooke}

School of Biological Sciences, University of Essex, Colchester, UK

Prostate cancer (PCa) cells grow in an environment which is known to cause endoplasmic reticulum (ER) stress. This leads to the activation of a process called the unfolded protein response (UPR) which tumour 
cells can utilise to survive and adapt to their adverse environmental conditions. Importantly, the UPR has also been shown to be directly regulated by the androgen receptor, linking the UPR to the key oncogenic driver of PCa. The aim of this project is to characterise UPR in PCa and to identify methods to target this pathway and promote tumour death. In order to mimic the stressful conditions of the tumour microenvironment, Tunicamycin - a natural occurring antibiotic, was used to induce ER stress in four PCa cell lines representing different stages of the disease (BPH-1, LNCaP, DU145 and PC3). Proliferation, as well as flow cytometry assays, were used to study the effects of ER stress upon PCa cell proliferation, cell cycle and cell death. Additionally, q-PCR analysis of downs-stream target genes was used to study the activation of the UPR in response to ER stress. It has been found that ER stress reduces the proliferation of all PCa cells and induces apoptosis. However, each cell line had a different sensitivity to ER stress. LNCaP cells were found to be the most sensitive, having all three UPR arms active after $6 \mathrm{~h}$ of stress and displaying G1 cell cycle arrest as well as high levels of apoptosis. DU145 had the highest apoptotic levels and the IRE1 $\alpha$ arm activated after $24 \mathrm{~h}$ of stress. BPH-1 and PC3 were less sensitive to ER stress, with BPH-1 having the lowest apoptotic levels and no UPR arms being activated in PC3 after $24 \mathrm{~h}$ of stress. Further research will investigate the effects of anti-androgens upon UPR signalling and characterise UPR target gene expression in response to Tunicamycin and UPR inhibitors. Finally, UPR signalling will be manipulated in order to identify novel therapeutic methods to treat therapy resistant disease.

\title{
39. Non-natural Macromolecules for new therapeutic strategies
}

\author{
Saeed Akkad ${ }^{1}$, Michelle D. Garrett ${ }^{2}$, Christopher J. Serpell ${ }^{1}$ \\ ${ }^{1}$ School of Physical Sciences, University of Kent, Canterbury, Kent CT2 7NH, UK \\ ${ }^{2}$ School of Biosciences, University of Kent, Canterbury, Kent CT2 7NJ, UK
}

At the heart of biology are macromolecules with high-precision architectures - proteins and nucleic acids which are integral to all processes of life. Medicine can be viewed as a mission to modulate the activity of these polymers. Historically, this has been achieved mainly using small molecules which bind to a specific region of a biomacromolecule. However, biochemistry is replete with macromolecule-on-macromolecule processes, pointing to alternative therapeutic strategies. To some extent this is reflected in the rise of biopharmaceuticals (biologics) as medicines, but this neglects the potentially much greater chemical space that is possible using non-natural macromolecules. Such species could be more highly tailored for certain purposes, possess a much greater range of functionalities, be tuned for localisation, degradation, and immunogenicity, and could be made in scalable processes as used for other chemical entities. We are using precision macromolecules to address cancer in two ways. In the first case, we are working to make the chemopreventive effects of non-steroidal anti-inflammatory drugs such as aspirin useful by creating nanocarriers which will direct them solely to cancer tissues. In the second approach, we are using a novel method to generate sequence-defined non-natural polymers which undergo programmed folding and self-assembly, analogous to proteins. These precision macromolecules are candidates for modulation of the most challenging oncogenic protein-protein interactions such as the RAS-RAF and MYC-MAX pairs. We are developing a chemical evolution strategy to identify binders of these proteins.

\section{A combination of CDK4/6 and IGF-IR inhibitors induces synergistic growth inhibition of human colorectal cancer cells with acquired resistance to anti-EGFR antibody}

\author{
S. A. Khelwatty ${ }^{1}$, S. Essapen ${ }^{1,2}$, A. M. Seddon ${ }^{1}$, Z. Fan ${ }^{3}$, H. Modjtahedi ${ }^{1}$ \\ ${ }^{1}$ Kingston University London, School of Life Science, London, UK \\ ${ }^{2}$ Royal Surrey County Hospital, St Luke's Cancer Centre, Guildford, UK \\ ${ }^{3}$ Department of Experimental Therapeutics, The University of Texas MD Anderson Cancer Center, Houston, \\ TX, USA
}


Acquired-resistance to anti-EGFR monoclonal antibodies (mAbs) is a major cause of treatment failure in patients with colorectal cancer (CRC), highlighting the need for more effective therapeutic strategies in such patients. We have previously shown that acquired-resistance to anti-EGFR mAb ICR62 in DiFi CRC cells (DiFi62) is accompanied by an increased EGFR expression, and augmented HER-2/HER-3 signalling and sensitivity to the pan-HER blocker afatinib. In this study, we investigated the therapeutic potential of the CDK inhibitors (palbociclib and ribociclib) as a single agent or in combination with the IGF-IR inhibitors (NVP-AEW541, NVP-ADW742) or MEK inhibitor (trametinib) in parental DiFi cells and their resistant varaints. The ICR62 resistant varaint was found to be highly resistant to treatment with palbociclib and trametinib. Interestingly, the gefitinib resistant DiFiG cells were found to be sensitive to NVPAEW541, NVP-ADW742, ribociclib, palbociclib and trametinib. While the combination of CDK inhibitors with NVP-AEW541 yielded synergistic growth inhibition in DiFi62 cells, the same combination was antagonistic in DiFiG cells. In addition, trametinib combined with either ribociclib or NVP-AEW541 yielded synergistic effect in both DiFi62 and DiFiG variants. Using proteome profiler kits, we found DiFiG cells had an increased phosphorylation of EGFR, insulin receptor, IGF-IR and ERK1/2, but HER-2 and HER-3 were undetectable. A significant increase in the levels of CDK4/6 was observed in DiFi62, but its level of expression was lower in the DiFiG variant. Moreover, treatment with CDK inhibitors or trametinib was able to entirely block the signalling of CDK4/6 and cyclin-D1 in DiFiG cells, but not in DiFi62 cells, suggesting that the mechanisms of acquired resistance to anti-EGFR mAb or TKI in these two variants are indeed different. Taken together, our results support further investigation on the therapeutic potential of combining CDK4/6 and IGF-IR inhibitors in CRC cells with acquired resistance to anti-EGFR mAb.

\title{
41. ATP-binding cassette - transporter upregulation; a potential mechanism of resistance to CDK7 inhibitors THZ1 and ICEC0942
}

\section{Georgina P. Sava, Charles Coombes, Laki Buluwela, Simak Ali}

\author{
Division of Cancer, Department of Surgery \& Cancer, Imperial College London, Hammersmith Hospital \\ Campus, London, UK
}

Cyclin-dependent kinase (CDK)7 is necessary for transcription initiation, via phosphorylation of the Cterminal domain of RNA polymerase II, and for cell cycle progression, through T-loop phosphorylation of the cell cycle CDKs. Recently it has been shown that CDK7 inhibition may be a useful novel approach in the treatment of multiple cancer types. We have developed a new, orally bioavailable selective CDK7 inhibitor, ICEC0942, that is currently being evaluated in a Phase I clinical trial for advanced solid malignancies. Drug resistance is currently a major problem facing cancer treatment and cancer cells are able to evade therapy via a multitude of mechanisms, therefore we sought to identify mechanisms by which cancer cells may become resistant to CDK7 inhibition. Through long-term drug exposure, we have developed MCF7 breast cancer cell lines that are resistant to both ICEC0942 and the covalent CDK7 inhibitor, THZ1. Importantly, whilst cells exposed to ICEC0942 develop resistance to both ICEC0942 and THZ1, cells cultured long-term with THZ1 retain sensitivity to ICEC0942. We have identified upregulation of multi-drug resistance ATP-binding cassette (ABC) transporters in the resistant cell lines. Specifically, the ICEC0942and THZ1-resistant cells have upregulation of $\mathrm{ABC}$ subfamily $\mathrm{B}$ member 1 (ABCB1) or MDR1 and $\mathrm{ABC}$ subfamily $G$ member 2 (ABCG2) or BCRP, respectively. Increased ABC-transporter mediated efflux activity was confirmed using flow cytometry and verapamil and novobiocin, specific inhibitors of $\mathrm{ABCB} 1$ and $\mathrm{ABCG} 2$, were able to rescue $\mathrm{CDK} 7$ inhibitor sensitivity in the relevant resistant cell lines, demonstrating the importance of $\mathrm{ABC}$-transporter activity in these resistance models. In conclusion, we have identified ABCB1 upregulation as a common mechanism of resistance to ICEC0942 and THZ1, and ABCG2 upregulation as a mechanism of resistance to THZ1. Overall, we hope that identifying potential mechanisms of CDK7 inhibitor resistance will help guide their future clinical use. 
42. Myosin II reactivation and cytoskeletal remodelling as a hallmark and a vulnerability in melanoma therapy resistance

\author{
Jose L. Orgaz ${ }^{1}$, Maria Romina Girotti ${ }^{2,3}$, Eva Crosas-Molist ${ }^{1}$, Amine Sadok ${ }^{4}$, Anna Perdrix-Rosell ${ }^{1,5}$, \\ Irene Rodriguez-Hernandez ${ }^{1}$, Silvia Mele ${ }^{1}$, Florencia Veigas ${ }^{2}$, Mirella Georgouli ${ }^{1}$, Victoria Bridgeman ${ }^{5}$, \\ Panagiotis Karagiannis ${ }^{1,6}$, Pahini Pandya ${ }^{1}$, Lena Boehme ${ }^{1}$, Matthew Smith ${ }^{3}$, Gabriela Gremel ${ }^{3}$, Rebec- \\ ca Lee ${ }^{3}$, Elena Galvani ${ }^{3}$, Franziska Baenke ${ }^{3}$, Paul C. Lorigan7, Fredrik Wallberg ${ }^{4}$, Amaya Viros $^{3}$, Ilaria \\ Malanchi $^{5}$, Sophia Karagiannis ${ }^{1}$, Richard Marais ${ }^{3}$, Victoria Sanz-Moreno ${ }^{1}$ \\ ${ }^{1}$ King's College London, London, UK \\ ${ }^{2}$ Consejo Nacional de Investigaciones Científicas y Técnicas (CONICET), Buenos Aires, Argentina \\ ${ }^{3}$ Cancer Research UK Manchester Institute, Manchester, UK \\ ${ }^{4}$ The Institute of Cancer Research, London, UK \\ ${ }^{5}$ The Francis Crick Institute, London, UK \\ ${ }^{6}$ University Hospital of Hamburg Eppendorf, Hamburg, Germany \\ ${ }^{7}$ The University of Manchester, Christie NHS Foundation Trust, Manchester, UK
}

Targeted and immunotherapies improve survival of a proportion of melanoma patients. However, lack of response or therapy resistance are persistent problems in melanoma management. In this study, we provide evidence that early adaptation to treatment and acquisition of resistance to MAPK inhibitors (MAPKi) involve profound cytoskeletal remodelling. This is concomitant with changes at the transcriptomic and phospho-proteomic level of many cytoskeletal proteins. Most significant changes are observed in the ROCKMyosin II pathway, widely studied for its key role in cancer invasion and metastasis. Furthermore, we find that MAPK signalling itself positively controls Myosin II activity. After initial therapy response, drugresistant clones restore MAPK and, in turn, Myosin II levels by increasing expression of myosin light chain 2, ROCK, LIM kinase and myocardin-related transcription factor. Cross-resistance to MAPKi and immune checkpoint inhibitors has been recently proposed to be controlled by common transcriptomic alterations. We report that high ROCK-Myosin II activity and its characteristic transcriptome identify both targetedand immunotherapy-resistant melanomas. Importantly, resistant cells are more dependent on Myosin II activity for their survival. ROCK1/2 or Myosin II activity blockade with either small molecule inhibitors or RNAi depletion induces death of resistant cells through a combination of cell cycle defects and decreased survival signals. Furthermore, efficacy of both targeted and immunotherapies can be greatly improved by combining such therapies with ROCK inhibitors in vivo. Therefore, we propose that a subset of targeted and immunotherapy-resistant melanomas is more vulnerable to ROCK-Myosin II inhibition, which opens up clinical opportunities for combination therapies. This work is currently under revision for Cancer Cell.

\title{
43. HIF-mediated suppression of DEPTOR confers resistance to mTOR kinase inhibition in renal cancer
}

\section{Jogitha Selvarajah, Shruthi Devkumar, Hong Doan, Alexander Parsons, Francesc Miralles, Ve- ronica A. Carroll}

\section{St George's, University of London, Cranmer Terrace, London SW17 oRE, UK}

Kidney cancer is diagnosed in over 300,000 individuals annually. Most kidney tumours are clear cell renal cell carcinomas (ccRCC) and metastatic disease is still largely incurable, despite some advances in targeted therapeutics. However, both intrinsic and acquired drug resistance are major causes of failure of targeted therapy in ccRCC. Mechanistic target of rapamycin (mTOR) is a fundamental regulator of cell growth and 
metabolism. The mTOR signalling pathway is upregulated in ccRCC and is an important therapeutic target, but some mTOR inhibitors do not work as expected, particularly second generation ATP-competitive mTOR kinase and dual PI3K/Akt/mTOR drugs. We have uncovered a resistance pathway to mTOR kinase inhibition which is mediated by DEP-domain containing mTOR-interacting protein (DEPTOR). DEPTOR is an endogenous inhibitor of mTOR and is critical for mTOR homeostasis and coordinated negative feedback control to PI3K/Akt. Here, we show that DEPTOR is strikingly suppressed in ccRCC tumours and cell lines. We demonstrate that DEPTOR is repressed by both hypoxia-inducible factors, HIF-1 and HIF-2, which are upregulated in renal cancer. DEPTOR knock-in and CRISPR/Cas9 mediated knock-out experiments show that DEPTOR is growth inhibitory in ccRCC. Furthermore, loss of DEPTOR confers resistance to second generation mTOR kinase inhibitors through deregulated mTORC1 feedback to PI3K/Akt. Our work reveals new mechanistic insight into deregulation of MTOR in ccRCC that is mediated by HIF-dependent reprograming of mTOR/DEPTOR networks. Importantly, we have identified a hitherto unknown mechanism of resistance to mTOR kinase targeted therapy in ccRCC. This work suggests that restoration of DEPTOR in ccRCC will confer sensitivity to mTOR kinase inhibition.

\title{
44. Acquired resistance to the experimental cancer drug capivasertib is associated with altered cap-dependent protein translation
}

\author{
Jasmine M. Jakubowski ${ }^{1}$, Denis T. Akan ${ }^{1,2}$, Mike I. Walton ${ }^{2}$, Michelle D. Garrett ${ }^{1}$ \\ ${ }^{1}$ School of Biosciences, University of Kent, Canterbury, UK \\ ${ }^{2}$ The Institute of Cancer Research, London, UK
}

The PI3K/AKT/mTOR (PAM) pathway is important for regulating cell growth, proliferation and survival in the cell and its deregulation is commonly observed in many cancers. For this reason, PAM inhibitors are undergoing development as a novel form of targeted cancer treatment. Since emergence of drug resistance is the major problem facing novel targeted therapies, our aim is to identify resistance mechanisms against the novel ATP-competitive AKT inhibitor capivasertib (AZD5363), which has currently completed Phase II clinical trials. The A2780 ovarian carcinoma cell line was used as a model to identify resistance mechanisms against capivasertib. A resistant subpopulation (5363R) was generated by dose escalation exposure to the drug and subcloned. These were investigated against the parental to identify molecular changes. We found that the A2780-5363R cells exhibit cross-resistance to both ATP-competitive (17.5 fold difference in $\mathrm{GI}_{50}$ ) and allosteric AKT inhibition (8.4 fold) and to inhibitors further downstream the PAM pathway. These include the rapalogue everolimus ( 78.7 fold) and the mTOR kinase inhibitor vistusertib (3.9 fold). As $4 \mathrm{EBP} 1$ phosphorylation is understood to be a rapalogue-resistant function of mTORC1 and coupled with a reduction of $4 \mathrm{EBP} 1$ in the resistant cells, $4 \mathrm{EBP} 1$ was identified as a potential candidate resistance mechanism. Pull-down of eIF4E showed reduced 4EBP1 and increased eIF4G binding to eIF4E in the 5363R cells, independent of mTOR inhibition thus suggesting an increase in cap-dependent protein translation. Protein synthesis analyses are consistent with this finding. These results show that increased protein translation is a mechanism of acquired resistance to AZD5363.

\section{FLIPi: evaluating a novel therapeutic strategy to target advanced prostate cancer}

\section{Daniel Turnham ${ }^{1}$, Norman Maitland ${ }^{2}$, Andrea Brancale ${ }^{3}$, Andrew Westwell ${ }^{3}$, Rhiannon French ${ }^{1}$, Richard Clarkson ${ }^{1}$}

${ }^{1}$ European Cancer Stem Cell Research Institute, School of Bioscience, Cardiff University, Cardiff, UK

${ }^{2}$ Department of Biology, University of York, York, UK

${ }^{3}$ School of Pharmacy, Cardiff University, Cardiff, UK 
Current treatment strategies for non-metastatic localised prostate cancer patients classified as "high risk" rely upon either radical prostatectomy or radiotherapy followed by androgen deprivation therapy. Despite these interventions, a significant proportion of patients with high-risk disease acquire resistance and progress with metastasis which will ultimately result in death. Increasing evidence suggests that a small population of tumour-initiating cells, cancer stem cells (CSCs), are responsible for this progression and are capable of evading, or are refractory to, standard therapies. Targeting CSCs therefore offers an interesting therapeutic strategy which should offer more sustained responses to treatment. We have recently shown that inhibiting the anti-apoptotic protein cFLIP in breast cancer cells dramatically sensitizes CSCs to the targeted apoptotic agent TRAIL, resulting in a profound decrease in experimental metastasis. cFLIP is overexpressed in localized high-grade prostate tumours, and as these tumours are associated with increased-risk of metastasis, we hypothesize that cFLIP may play a similar role in the maintenance of CSCs in prostate cancers. Using a novel pharmacological inhibitor of cFLIP (OH14) developed in our laboratory we have confirmed that cFLIP suppression combined with TRAIL treatment selectively targets prostate CSCs in both established and patient derived cultures. Furthermore, OH14 alone has shown efficacy as a monotherapy at reducing bulk cell viability in low grade primary prostate cancers. Interestingly, we have found cFLIP to be overexpressed in the metastatic subline PC-3M, with OH14 and TRAIL treatment significantly reducing bulk and CSC activity in these cells. We are now utilising orthotopic models of metastatic disease as well as developing a variety of PDX models to investigate how this combination acts in vivo whilst also investigating the role of cFLIP in models of acquired resistance to both docetaxel and enzalutamide in vitro. We believe that this CSC-based therapeutic intervention may have the potential to halt the spread of disease and prevent recurrence in high-risk patients, which no current treatment option is able to do.

\title{
46. Chronic Myeloid Leukaemia Resistance to Therapy: running on RUNX1
}

\section{Naomi Gibson, Geetha Bheeshmachar, Peter Laslo}

Faculty of Medicine and Health, University of Leeds, St James's Hospital, Leeds, UK

Chronic Myeloid Leukaemia (CML) is associated with the BCR-ABL1 (BA) tyrosine kinase oncogene and managed with drug therapy [Imatinib (IM)]. However, $20 \%$ of patients develop drug resistance, resulting in fatality within 18 months. A preclinical model of IM resistance using a CML cell line (KCL-22 cells) established that IM resistant (IMr) cells are kinase independent, recapitulating clinical observations where treatment inhibits BA activity yet the cells continue to survive. Therefore, the IMr cells must acquire new oncogenic drivers that compensate for the loss of BA activity. Gene expression analysis revealed that $>1,400$ genes are differentially regulated in IMr cells. Among these genes, the haematopoietic transcription factor RUNX1 was of particular interest, being dysregulated in multiple haematological malignancies. RUNX1 expression is upregulated upon IM resistance in CML patients and our pre-clinical model, and RUNX1 protein is highly induced in the IMr cells. Knockdown of RUNX1 using two different siRNA constructs caused growth inhibition of IMr cells. Further analysis revealed that RUNX1 depletion induced a macrophage-like morphology of IMr cells and the upregulation of myeloid-associated genes including CD11b and MCSF-R, suggesting that RUNX1 depletion results in partial differentiation of IMr cells. Pharmacological inhibition of RUNX1 activity replicated these results, highlighting its potential as a therapeutic target.

\section{Downregulation of SLFN11 confers resistance to DNA damaging agents; insights into resensitization with novel DNA damage response inhibitor combinations}

\author{
Claudia Winkler', Tarrion Baird ${ }^{2}$, Michael P. Menden ${ }^{1}$, Jenni Nikkilä', Martine P. Roudier ${ }^{2}$, \\ Gemma N. Jones ${ }^{2}$, Andrew J. Pierce ${ }^{2}$, Mark O' Connor ${ }^{1}$, Elisabetta Leo ${ }^{1}$
}


${ }^{1}$ Oncology Bioscience, IMED Biotech Unit, AstraZeneca, Cambridge, UK

${ }^{2}$ Oncology Translational sciences, IMED Biotech Unit, AstraZeneca, Cambridge, UK

Schlafen 11 (SLFN11) is a putative DNA/RNA helicase only found in humans and some primates, with bimodal distribution in cancer cells. It was initially discovered by bioinformatics analyses of cancer cell databases as a genomic determinant of response to various DNA damaging agents (DDA). Most importantly, recent reports indicate that SLFN11 expression in tumours may serve as a predictive biomarker for stratification of cancer patients treated with DDA and DNA damage response inhibitor (DDRi). In the Sanger panel of cancer cell lines, we independently validated SLFN11 downregulation in $\sim 50 \%$ of cells, and that its expression correlates with response to DDA as well as PARP inhibitors. To further shed light on SLFN11 mechanism of action we knocked out SLFN11 (KO) in prostate cancer cells DU145 via CRISPR-Cas9. Relevant clones were selected and the isogenic pair was profiled for SLFN11 downregulation at protein levels (by western blotting and immunofluorescence) and were instrumental for the development of an FFPE immunohistochemistry (IHC) assay. This was further implemented and validated in xenograft tissue and was found to be specific. The susceptibility of SLFN11 KO cells to different DDAs and DDRi was verified by cell viability assays. Downregulation of SLFN11 conferred resistance to treatments with the topoisomerase inhibitors ETP and camptothecin (CPT), as well as to various DDRi. Interestingly, combinations of ETP with some DDRi were found to be more synergistic in SLFN11 KO than in wild type cells, suggesting that these combinations could be more effective in cells that lack SLFN11. A detailed analysis of these results will be presented here and indicate that selective DDA-DDRi strategies may re-sensitize cancers that have become resistant to chemotherapy due to SLFN11 downregulation.

\title{
48. Changing faces of FUS: FUS/TLS represses androgen receptor activity in prostate cancer through disruption of the transcriptional complex
}

\author{
G. N. Brooke ${ }^{1,2}$, R. C. Culley ${ }^{1}$, F. M. Fioretti ${ }^{1}$, C. Reader ${ }^{1}$, E. Rees ${ }^{2}$, S. M. Powell ${ }^{1}$, M. Alkheilewi ${ }^{2}$, \\ A. Pine ${ }^{2}$, L. Latonen ${ }^{3}$, T. Visakorpi ${ }^{3}$, L. Gaughan ${ }^{4}$, D. A. Leach1, J. Waxman', C. L. Bevan ${ }^{1}$ \\ ${ }^{1}$ Imperial College, London, UK \\ ${ }^{2}$ University of Essex, Colchester, UK \\ ${ }^{3}$ University of Tampere, Finland \\ ${ }^{4}$ Newcastle University, UK
}

Prostate cancer growth is dependent upon the androgen receptor, a ligand-dependent transcription factor which exerts its effects on transcription in concert with cofactor proteins (coactivators and corepressors). We have previously shown that FUS/TLS, a multi-functional protein, is down-regulated in response to androgen receptor signalling and that it has tumour suppressor properties. To better understand what FUS regulates to block tumour growth, the transcriptome of the androgen-dependent prostate cancer cell line LNCaP was investigated in response to FUS over-expression. This analysis identified a high degree of overlap between FUS-regulated genes and those regulated by the androgen receptor. Further, FUS was found to predominantly repress androgen receptor activity and this was confirmed using reporter assays. Mammalian 2-hybrid and trans-repression assays demonstrated that FUS interacts with the androgen receptor via its RNA recognition motif domain and contains both transrepression and activation domains. Interestingly, the transrepression domains are HDAC independent and do not contribute to the repressive action of FUS upon the androgen receptor. Instead, it appears that FUS represses androgen receptor activity as a result of competition for coactivators. A modified mammalian 2-hybrid assay demonstrated that the N-terminal activation function of FUS interacts with coactivators such as SRC1, and chromatin immunoprecipitation studies demonstrated that FUS inhibits the formation of the active androgen receptor transcriptional complex. In agreement with our previous findings, FUS levels were reduced in prostate cancer, but interestingly were elevated in the aggressive, castrate resistant stage of the disease. It therefore appears 
that FUS is down-regulated in prostate cancer, reducing the levels of an androgen receptor repressor. In contrast, FUS levels are increased in advanced therapy resistant prostate cancer, suggesting that it may play a different role in this late stage of the disease.

\title{
49. Targeting phospholipase $\mathrm{C}$ as a novel therapeutic option for the treatment of prostate cancer
}

\author{
Mohammad Alkheilewi ${ }^{1}$, Andrea Mohr ${ }^{1}$, Ralf Zwacka ${ }^{1}$, Metodi Metodiev ${ }^{1}$, Euphemia Leung ${ }^{2}$, \\ Michelle Van Rensburg ${ }^{2}$, David Barker ${ }^{2}$, Johannes Reynisson ${ }^{2}$, Greg Brooke ${ }^{1}$
}

\author{
${ }^{1}$ Univeristy of Essex, Colchester, UK \\ ${ }^{2}$ University of Auckland, New Zealand
}

Prostate cancer (PCa) growth is driven by the androgen receptor and hence therapies often target this signalling axis. Although successful initially, the treatments invariably fail and the tumours progress to the aggressive castrate resistant stage of the disease. There is therefore a great need for novel therapeutics for castrate resistant disease. Thieno[2,3-b] pyridines anticancer compounds were discovered by virtual high throughput screening as potential inhibitors of phospholipase $\mathrm{C}$, which has been shown to promote tumour growth and metastasis. These compounds have been previously demonstrated to be effective inhibitors of breast cancer. Here we expanded the research by investigating the efficacy of these compounds on PCa cell lines. Using growth assays, flow-cytometry analysis, florescent imaging and cell tracking assays I have demonstrated that the compounds are potent inhibitors of PCa proliferation and motility. Importantly, some of the compounds show less activity in the non-tumorigenic control BPH1, suggesting that these drugs are more specific for cancer cells. Interestingly, the drugs were also found to induce multi-nucleation, $\mathrm{G}_{2} / \mathrm{M}$ arrest and promote apoptosis. In conclusion, these compounds represent a novel therapeutic approach for PCa and further work to assess their efficacy is warranted.

\section{Nanodelivery of CYP26 inhibitors enhances the actions of retinoic acid in neuroblastoma-derived tumour cells}

\author{
Shreya Shah ${ }^{1}$, Maja Bilip ${ }^{1}$, Mayuran Mathiyalakan ${ }^{1}$, Aris Tagalakis ${ }^{1}$, Stephen Hart ${ }^{1}$, \\ Claire Simons ${ }^{2}$, Andrew W. Stoker ${ }^{1}$ \\ ${ }^{1}$ UCL Great Ormond Street Institute of Child Health, 30 Guilford Street, London WC1N 1EH, UK \\ ${ }^{2}$ School of Pharmacy and Pharmaceutical Sciences, Cardiff University, Cardiff, UK
}

Neuroblastoma is a paediatric, sympathetic nervous system cancer in which the five-year survival of high grade tumour patients remains poor. After multimodal treatments, patients receive residual disease therapy with retinoic acid (RA), a natural, vitamin A-derived neural differentiation agent. While RA can modestly improve survival, it is beset with challenges of effective dosing, toxicity and evolved resistance. RA in particular suffers poor pharmacokinetics due to its rapid metabolism by CYP26 enzymes and these enzymes are also thought to be responsible for some forms of RA resistance. RA efficacy might thus be improved through CYP26 inhibition in tumour cells. Given the highly hydrophobic nature of many CYP26 inhibitors, we also hypothesized that inhibitor bioavailability may be further improved through liposomal nanodelivery. Several RA metabolism-blocking agents (RAMBAs) exist as inhibitors of CYP26 enzymes, but these have not so far been tested for their abilities to either increase neuroblastoma cell differentiation, or enhance the suppression of cell growth in response to RA. Here we demonstrate that the bioavailability of these hydrophobic RAMBAs can be facilitated using liposomal nanoparticles. In RA-treated neuroblastoma cell lines, we show that both ethanol-solubilized and liposome-loaded RAMBAs can successfully 
enhance the actions of RA, generating increased cell differentiation, suppression of cell proliferation and suppression of the neuroblastoma driver protein N-Myc. We propose that the nanodelivery of RAMBAs may provide a new approach for effective enhancement of RA action in neuroblastoma, potentially reversing some forms of CYP26-based RA resistance, and facilitating more selective tumour targeting of these agents.

\title{
51. Acquisition of a mesenchymal phenotype in endometrial cancer through epigenetic manipulation may contribute to therapeutic resistance
}

\author{
L. D. Ordonez ${ }^{1}$, L. Blake ${ }^{1}$, L. Margarit ${ }^{2}$, D. Gonzalez ${ }^{1}$, P. Rees ${ }^{3}$, L. Francis ${ }^{1}$, R. S. Conlan ${ }^{1}$ \\ ${ }^{1}$ Reproductive Biology and Gynaecological Oncology Group, Swansea University Medical School \\ ${ }^{2}$ Abertawe Bro Morgannwg University Health Board \\ ${ }^{3}$ College of Engineering, Swansea University
}

Endometrial cancer (EC) is the most common gynecological cancer and the fourth most common cancer in females in the UK. Classified into two histotypes, Type I correlates with estrogen receptor-positivity (ER) and good prognosis while Type II, presents as ER-negative, high-grade disease with poor prognosis. Despite advances in treatment, intrinsic and acquired resistance is a major problem, hindering the effectiveness of drugs in the clinic. A better understanding of the mechanisms that determines resistance could make a significant impact. To investigate the role of epigenetic instability in driving cellular changes in EC we utilized cell lines to model Type I (Ishikawa) and Type II (Hec50) treated in vitro with the de-methylation drug 5-Aza-Cdr. Ishikawa were more sensitive to treatment than Hec50. Hec50 cells showed a higher proportion of cells with mesenchymal morphology and sublethal doses of 5-Aza-Cdr for 96 hours resulted in an increase in mesenchymal cells in both cell lines. These changes correlated with a decrease in E-cadherin in Ishikawa and increase in $\mathrm{N}$-cadherin in Hec50, suggesting partial epithelial-to-mesenchymal transition (EMT). EMT has previously been shown to correlate with invasion and treated cells showed promoter demethylation of genes that contribute to invasion (MMP9, MMP12, CXCL12) in both cell lines, correlating with biophysical alterations suggestive of increased cellular motility. Treated Hec50 were cultured for a further four weeks and showed the mesenchymal population was the most prominent to remain, suggesting that this population could contribute to resistance. In conclusion our work suggests that a mesenchymal phenotype may be involved in intrinsic and acquired resistance to therapy in EC, with a subpopulation of cells acquiring a partial transition, as a result of epigenetic manipulation, during early stages of treatment. Such mechanisms, well documented in breast cancer, are targets for next generation drugs, which may present advantageous therapeutic strategies in the uterus.

\section{By-pass of irinotecan resistance by the potent topoisomerase I inhibitor LMP400}

\section{Jan Stenvang ${ }^{1}$, Yves Pommier², Niels Frank Jensen ${ }^{1}$, Mark Cushman ${ }^{3}$, Mark Roger $^{4}$, Nils Brünner ${ }^{1}$}

\author{
${ }^{1}$ University of Copenhagen, Institute of Drug Design and Pharmacology \& Scandion Oncology, Copenhagen, \\ Denmark \\ ${ }^{2}$ Development Therapeutics Branch and laboratory for Molecular Pharmacology, Center for Cancer Research, \\ National Cancer Institute, NIH, Bethesda, MD, USA \\ ${ }^{3}$ Department of Medicinal Chemistry and Molecular Pharmacology, and The Purdue Center for Cancer Re- \\ search, Purdue University, West Lafayette, Indiana 47907, USA \\ ${ }^{4}$ Gibson Oncology, Miami, FL 33109, USA
}


Irinotecan is a topoisomerase I inhibitor used to treat patients with metastatic colorectal cancer (CRC). However, although approximately $50 \%$ of these patients will initially benefit from first-line irinotecancontaining treatment, they will all experience progressive disease. We developed pairs of SN38-sensitive and SN38-resistant human CRC cell lines (Jensen et al., Mol. Oncol, 2015). Two of three cell lines had significant upregulation of ABCG2 and inhibition of this drug efflux pump with a synthetic inhibitor (Ko143) abolished SN38 resistance in ABCG2-upregulated cell lines. An alternative to using pump inhibitors to block topoisomerase I inhibitor resistance is to use topoisomerase I inhibitors that are not substrates for ABCG2. LMP400, which belongs to the indenoisoquinoline family, was identified from the NCI60 data files as a potent topoisomerase I inhibitor. We exposed ABCG2-upregulated SN38-resistant cell lines to LMP400 and observed a highly significant anti-tumor effect indicating that LMP400 is not a substrate for the ABCG2 efflux pump. Another notable feature of LMP400 is that it - in contrast to SN38 - is not metabolized by UGT-1A1 and it is therefore not expected to result in severe side effects in patients with mutated UGT-1A1. Finally, LMP400 was also active in colorectal cancer cell lines with acquired oxaliplatin resistance. LMP400 has passed clinical phase I studies in various cancer types with incurable disease (Kummar et al., Cancer Chemotherapy Pharmacol, 2016). The recommended dose for phase II studies is weekly 90 $\mathrm{mg} / \mathrm{m}^{2}$ with myelosuppression as the primary toxicity and with only limited GI side effects. We will initiate a study in patients with metastatic CRC progressing on irinotecan-containing chemotherapy. We will follow Simons two stage design with $\geq 2$ responders among 15 patients (RECIST version 1.1) being sufficient to include an additional 10 patients.

\section{SCO-101 is a first-in-class drug that reverses anti-estrogen resistance in breast cancer cells}

\section{Katrine Hartfelt ${ }^{1}$, Ida Christina Barner Madsen ${ }^{1}$, Signe Lykke Nielsen ${ }^{1}$, Palle Christophersen ${ }^{2}$, Nils Brünner ${ }^{1,3}$, Jan Stenvang ${ }^{1,3}$}

${ }^{1}$ Department of Drug Design and Pharmacology, Faculty of Health and Medical Sciences, University of Copenhagen, Jagtvej 160, Copenhagen DK-2100, Denmark

${ }^{2}$ Saniona, Baltorpvej 154, Ballerup DK-2750, Denmark

${ }^{3}$ Scandion Oncology, COBIS, Ole Maaløesvej 3, Copenhagen DK-2200, Denmark

Eighty percent of all cases of breast cancer patients present with estrogen receptor (ER)-positive disease and treatment with anti-estrogens is an effective treatment for these patients. However, anti-estrogen resistance uniformly arises in patients with advanced breast cancer. SCO-101, which is an inhibitor of the volume regulated anion channel (VRAC), is an oral drug, which exhibits a safe toxicology profile as demonstrated in four phase I clinical trials in healthy individuals. We investigated the potential of SCO-101 to act in combination with the anti-estrogens tamoxifen and fulvestrant in anti-estrogen resistant human breast cancer cell lines (MCF-7/LCC-2, MCF-7/LCC-9, T47D/TR1) and the ER negative MDA-MB-231. Treatment effects were investigated by MTT cell viability assays, siRNA knock-down experiments and western blots. SCO-101 only had minor inhibitory effects on cell viability of the cell lines when administered alone. However, when combining SCO-101 with tamoxifen or fulvestrant in MCF-7 or T47D antiestrogen resistant breast cancer cells, an additive to synergistic effect on cell viability was observed. Thus, SCO-101 re-sensitized the anti-estrogen resistant LCC-2, LCC-9 and T47D/TR1 cell lines to tamoxifen and fulvestrant. In contrast, SCO-101 in combination with anti-estrogens had no effects on the triple negative MDA-MB-231 cell line. As SCO-101 mainly targets VRAC, in which LRRC8A is the essential subunit, we investigated whether knockdown of LRRC $8 \mathrm{~A}$ would impact the treatment outcome. No apparent changes in response to SCO-101 and anti-estrogens were observed upon knockdown of LRRC8A. Additionally, the protein level of LRRC8A was examined upon treatment with anti-estrogens $+/$ - SCO-101 and the treatment did not alter LRRC8A protein expression. Our findings suggest that SCO-101 interferes with anti-estrogen 
resistance in breast cancer. However, more studies are needed before taking SCO-101 into clinical testing in patients with anti-estrogen resistant ER positive breast cancer.

\section{SCO-101 - an oral drug that interferes with drug resistance mechanisms in cancer}

\section{Jan Stenvang ${ }^{1}$, Palle Christophersen ${ }^{2}$, Nils Brünner ${ }^{1}$}

${ }^{1}$ Scandion Oncology A/S, Ole Maaløvs Vej 3, Copenhagen 2200, Denmark; and University of Copenhagen, Institute of Drug Design and Pharmacology, Copenhagen, Denmark

${ }^{2}$ Saniona A/S, Baltorpvej 154, Ballerup DK-2750, Denmark

SCO-101 is a volume regulated anion channel modulator initially developed to treat patients with sickle cell anemia (SCA). The drug in the form of an oral capsule has successfully passed 4 clinical phase I studies in healthy volunteers $(n=92)$. The studies included different genders, single and multiple dose escalation studies and potential influence of simultaneous food intake. The drug was safe with very limited toxicity (slight and reversible increase in serum bilirubin). Pharmacokinetic studies showed a T1/2 of $15 \mathrm{~h}$ and Cmax on day 1 of $10 \mu \mathrm{g} / \mathrm{mL}=20 \mu \mathrm{mol} / \mathrm{L}$ and at day 14 of $20 \mu \mathrm{g} / \mathrm{mL}=40 \mu \mathrm{mol} / \mathrm{L}$. Recommended dose for phase II was between 100-150 $\mathrm{mg} / \mathrm{kg}$. However, due to the increase in serum bilirubin, further development for SCA was stopped. SCO-101 significantly increases the effects of certain forms of chemotherapy in xenotransplanted human cancers. Moreover, SCO-101 reverts resistance to certain types of chemotherapy and antiestrogens. One recently discovered mechanism of action (MoA) of SCO-101 is inhibition of drug efflux pumps. Another MoA relates to inhibition of an intracellular signaling pathway being involved in drug resistance. Scandion Oncology will initiate clinical phase II studies with breast and colorectal cancer patients, consisting of a run-in part with SCO-101 dose escalations together with standard dose of anticancer therapy followed by a regular phase II study, which will follow Simons two stage design. For the chemotherapy studies, patients will be treated with one daily oral SCO-101 dose for one week. In week 2, patients will receive one dose of chemotherapy while continuing daily SCO-101 oral treatment. In week 3, there will be a drug holiday. These three weeks will constitute one treatment cycle. After two and four cycles, patients will be evaluated for objective response according to RECIST version 1.1. In addition, we will record progression-free survival and overall survival.

\section{The generation of models of acquired resistance using in vitro and in vivo techniques}

\section{Steve Wagner, Christopher Hindley, Keisha Hearn, Aurelie Courtin, Tomoko Smyth, Joanne Munck, Nicola Wallis}

Astex Pharmaceuticals, Cambridge, UK

The development of drug resistance against clinically approved targeted agents remains a major challenge for the treatment of various cancers, with patients frequently relapsing after initial response through multiple mechanisms of resistance. In order to identify and target molecules to overcome resistance to clinically approved drugs, better models of resistance are required. We have established multiple resistance models to targeted agents using a number of different techniques. Resistance was generated both in vitro and in vivo in order to mimic the development of acquired resistance in the patient. In vitro resistance was generated to targeted agents such as vemurafenib and erlotinib by chronic exposure of cancer cell lines driven by BRAF (A375, melanoma) and EGFR (HCC827, lung) to these agents over periods of months. Whilst the generation of resistance to some agents was relatively straight forward, other models required more optimisation and for some agents we struggled to establish a model of resistance in vitro. In vivo resistance 
was generated by dosing $\mathrm{H} 2228$ and HCC 827 xenograft bearing mice with the tyrosine kinase inhibitors, crizotinib and erlotinib respectively, for prolonged periods of time and subsequently culturing resistant tumours ex vivo. The cell lines resulting from both these types of model were the object of detailed analysis such as exome sequencing, RNA-Seq and protein analysis to establish mechanisms of resistance. These cell lines were subsequently used to test the ability of compounds to overcome resistance both in vitro and in vivo. In summary, our data exemplify how different techniques can be employed to study drug resistance in vitro as well as in vivo and to identify novel vulnerabilities in cancer cells caused by different resistance mechanisms.

\title{
56. Modelling stromal/cancer cell invasion as a platform to assess stromal targeted therapy
}

\author{
E P. Carter, A S. Wilson, H M. Kocher, R P. Grose
}

Centre of Tumour Biology, Barts Cancer Institute, Queen Mary University of London, London EC1M 6BQ, UK

Cancer cells co-opt their surrounding stroma to facilitate invasion. This is particularly evident in pancreatic cancer, which is characterised by a dense stroma largely comprised of activated stellate cells. 3D modelling presents an excellent method to analyse cancer/stromal interactions and the effects of potential stroma-targeted therapies. However, many of the current models are time-consuming, low-throughput, and require extensive processing for analysis. We have developed a fast, semi-high-throughput cancer/stromal invasion assay that can be used to dissect cancer/stromal interactions and quantitatively assess therapeutic drug action. Pancreatic cancer cells (Miapaca2) are combined with stellate cells (PS1) in spheroids, which are subsequently embedded in Matrigel matrix on glass in a 96 well plate format. Over $48 \mathrm{~h}$ dramatic cell invasion is observed from the central spheroid, with stellate cells leading the collective invasion of cancer cells. This is apparent from the confocal imaging of stellate and cancer cell markers, plus the use of fluorescently labelled cells. Measurement of the invasive area serves as a rapid quantitative readout. Fibroblast growth factor receptor 1 (FGFR1) activity in stellate cells has been reported to influence cancer cell invasion, and indeed, treatment with the FGFR inhibitor AZD4547 reduces invasion in a concentration-dependent manner. Thus, this model provides a fast, semi-high-throughput system to interrogate molecular crosstalk during cancer cell invasion and objectively interrogate both mechanism and efficacy of stromaltargeted therapies.

\section{Chemosensitivity associated chromatin conformation changes influence genomic cisplatin-adduct distribution}

\author{
John Gallon', Erick Loomis ${ }^{1}$, Nicholas Martin², James Flanagan ${ }^{1}$, Robert Brown ${ }^{1}$ \\ ${ }^{1}$ Department of Surgery and Cancer, Imperial College London, London, UK \\ ${ }^{2}$ Trace Element Laboratory, Clinical Biochemistry, Charing Cross Hospital
}

Changes in chromatin organization are associated with sensitivity/resistance to DNA damaging, anticancer drugs, such as cisplatin; widely used in the treatment of ovarian cancer. However, how chromatin conformation changes during the acquisition of resistance and how this influences cisplatin-DNA adduct formation are poorly understood. We investigated platinum-adduct distribution in the context of chromatin accessibility in three pairs of cisplatin sensitive and resistant ovarian cancer cell lines, including lines isolated from high-grade serous ovarian cancer patients following chemotherapy (PEO1-PEO4). Using ATAC-seq we show chromatin accessibility changes distinguishing resistant ovarian cell lines from their sensitive counterparts, with regions of differentially accessible (DA) chromatin primarily at intergenic 
loci. In the A2780-CP70 and PEO1-PEO4 pairs, DA regions were predominantly less accessible in resistant lines while equal doses of cisplatin induced fewer platinum-adducts in the resistant line, as shown by inductively-coupled plasma mass spectrometry. Using a novel assay, Pt-ExoSeq, analysis of genome wide platinum-adduct distribution distinguished sensitive PEO1 from its resistant counterpart PEO4, when treated with doses inducing equal total damage in both lines. Chromatin conformation changes associated with resistance influence adduct formation. Thus, we observe reduced adduct formation in $\mathrm{PEO} 4$ compared to PEO1 at significantly less accessible genomic regions, primarily around gene promoters. However, a set of intergenic regions with increased damage in $\mathrm{PEO} 4$ show a larger reduction in accessibility. To confirm that greater accessibility increases cisplatin-adduct formation, we increased histone acetylation and chromatin accessibility using the histone deacetylase inhibitor Vorinostat, before treating with cisplatin. This led to greatly increased adduct formation. We show for the first time that platinum-adduct distribution and chromatin accessibility analysed genome-wide, can differentiate platinum sensitive and resistant ovarian cell lines and suggest a role for chromatin conformation changes in the emergence of drug resistance by altering the distribution of platinum-DNA adducts throughout the genome and hence their cytotoxic effects.

\title{
58. Increased ERK phosphorylation as a candidate driver of resistance to the experimental cancer drug AT13148
}

\author{
Nathan D. Breeds ${ }^{1}$, Denis Akan ${ }^{1,2}$, Mike I. Walton ${ }^{2}$, Michelle D. Garrett ${ }^{1,2}$ \\ ${ }^{1}$ University of Kent, School of Biosciences, Canterbury, UK \\ ${ }^{2}$ The Institute of Cancer Research, CRUK Cancer Therapeutics Unit, London, UK
}

The AGC family of serine/threonine protein kinases comprises a number of drug targets with therapeutic potential for the treatment of cancer. AT13148 is an ATP competitive AGC kinase inhibitor, currently in phase 1 clinical trials, with activity against several members of the AGC kinase family, including ROCK $1 / 2$, AKT, and p70S6K. Due to the prevalence of acquired drug resistance in the clinic, elucidating mechanisms of acquired resistance early in the development of an experimental cancer drug may provide opportunities to overcome resistance. The aim of this project was therefore to develop preclinical models of AT13148 resistance and identify potential driver mechanisms prior to clinical emergence. Isogenic AT13148 resistant sub-clones were generated from the A2780 ovarian carcinoma cell line. These displayed increased ERK 1/2 phosphorylation concurrent with decreased MEK 1/2 phosphorylation, when compared to the parental A2780 cell line. In addition, two of the resistant sub-clones were sensitised to AT13148 on exposure to the ERK inhibitor GDC-0994, implicating ERK as a driver of resistance. These sub-clones also exhibited cross-resistance to MEK inhibitors (PD-0325901 and selumetinib), with ERK phosphorylation shown to be refractory to PD-0325901. Loss of DUSP 6 expression, an ERK 1/2 phosphatase, was subsequently detected in these AT13148 resistant sub-clones and is hypothesised as a potential mechanism of increased ERK phosphorylation and therefore resistance.

\section{Identification of candidate genes that may function in oestrogen receptor - breast cancer}

\section{Stephen Chandler ${ }^{1}$, Francesco Crea ${ }^{1}$, Mark Hirst ${ }^{1}$, Angela Cox ${ }^{2}$, Sushilaben Rigas ${ }^{1}$}

${ }^{1}$ The Open University, Milton Keynes, UK

${ }^{2}$ University of Sheffield, Sheffield, UK

Breast cancer is the most common cancer in women worldwide. Most breast cancers are hormone related involving the oestrogen receptor (ER), progesterone receptor (PR) and/or the human epidermal growth 
factor (HER2). The majority of aggressive (invasive and metastatic) breast cancers are negative for the ER receptor (ER-) including the triple negative and basal subtypes (ER-, PR-, HER2-). Due to this, traditional treatments such as hormonal replacement therapy are not suitable. Using bioinformatics, $\mathrm{R}$ scripting, and datasets from 1,485 Caucasian samples (1,437 tumours and 48 normal tissues) with 21,000 genes from the publicly available online GEO database (NCBI), we compared expression levels of ER negative (ER-) tumours to ER positive (ER+) tumours. 7 protein coding genes were identified as candidate functional genes, where their expression was significantly up-regulated in ER- tumours $\left(\log 2 \mathrm{FC}=1.02-1.24, P=2.98 \mathrm{E}^{-31}\right.$ $6.47 \mathrm{E}^{-19}$ ) which correlated with reduced survival using distant metastasis free survival in Kaplan-Meier plotter (Log rank test, $P=0.0013-0.033$ ). Next, we will determine the effect of copy number changes, the functions of the genes in triple negative breast cancers and in cellular pathways, and then validate these results in the laboratory to test their potential as novel therapeutic targets in aggressive cancers and as biomarkers to predict prognosis or metastases.

\title{
60. CRUK-Medlmmune Alliance Laboratory: a unique partnership in the discovery and development of novel biologics
}

\section{Sebastian Fielder, Denice Chan}

Cancer Research UK-MedImmune Alliance Laboratory, The Portway Building, Granta Pk, Cambridge CB21 $6 G S, U K$

In September 2014 Cancer Research UK (CRUK) and MedImmune entered into an innovative collaboration with the aim of setting up a laboratory in Cambridge to develop novel cancer drugs for patients. As part of its research review, CRUK identified that it needed to better leverage its biological expertise and play a more prominent role in biotherapeutic discovery. At the same time, MedImmune were driving forward towards the potential of more cures and realized the role key collaborations with organizations like CRUK could play in the generation of novel oncology therapeutics. Crucial to the success of the Alliance is for the lab to receive target proposals from researchers across the UK with and without CRUK funding. Our mission: (1) to maximise cancer patient benefit; (2) to run a collaborative research laboratory; (3) to actively engage the oncology research community; (4) to partner closely with investigators.

\section{Using tumour deconvolution to identify determinants of anti-tumour immunity and checkpoint blockade response}

\section{Ankur Chakravarthy ${ }^{1}$, Andrew Furness ${ }^{2}$, Kroopa Joshi ${ }^{2}$, Ehsan Ghorani ${ }^{2}$, Kirsty Ford ${ }^{3}$, Matthew J. Ward ${ }^{3}$, Emma V. King ${ }^{3}$, Matt Lechner ${ }^{1}$, Teresa Marafioti ${ }^{4}$, Sergio A. Quezada ${ }^{2}$, Gareth J. Thomas $^{3}$, Andrew Feber ${ }^{5}$, Tim R. Fenton ${ }^{6}$}

\author{
${ }^{1}$ Department of Oncology, UCL Cancer Institute, University College London, London WC1E 6BT, UK; present \\ address: Princess Margaret Cancer Centre, Toronto, Ontario M5G 2C4, Canada \\ ${ }^{2}$ Department of Haematology, UCL Cancer Institute, University College London, London WC1E 6BT, UK \\ ${ }^{3}$ Cancer Sciences Unit, University of Southampton, Tremona Road, Southampton SO16 6YD, UK \\ ${ }^{4}$ Department of Pathology, UCL Cancer Institute, University College London, London WC1E 6BT, UK \\ ${ }^{5}$ Division of Surgery and Interventional Science, University College London, London WC1E 6BT, UK \\ ${ }^{6}$ School of Biosciences, University of Kent, Canterbury CT2 7NJ, UK
}

The nature and extent of immune cell infiltration into solid tumours are key determinants of therapeutic response. Using a DNA methylation-based approach to tumour cell fraction deconvolution, we report the 
integrated analysis of tumour composition and genomics across a wide spectrum of solid cancers profiled by The Cancer Genome Atlas Project. Initially studying head and neck squamous cell carcinoma, we identify two distinct tumour subgroups: "immune hot" and "immune cold", which display differing mutation burden, cytokine signalling, cytolytic activity, and oncogenic driver events. We demonstrate the existence of such tumour subgroups across 18 further solid cancer types and, using gene expression data from recent clinical trials, show that transcriptional signatures of hot tumours are selectively engaged in melanomas that respond to immune checkpoint blockade. Finally, we show that treatment-naive hot tumours are markedly enriched for known immune-resistance genomic alterations, potentially explaining the heterogeneity of immunotherapy response and prognosis seen within this group. Finally, our genomic copy number analysis revealed potential targets for "warming-up" cold tumours, including epidermal growth factor receptor signalling.

\title{
62. Quantitative live imaging of Ponatinib in vitro enabled by stimulated Raman scattering microscopy
}

\author{
Kristel Sepp ${ }^{1,2}$, Martin Lee ${ }^{2}$, Valerie G. Brunton ${ }^{2}$, Alison N. Hulme ${ }^{1}$ \\ ${ }^{1}$ EaStCHEM School of Chemistry, The University of Edinburgh, Joseph Black Building, David Brewster Road, \\ Edinburgh EH9 3FJ, UK \\ ${ }^{2}$ Edinburgh Cancer Research Centre, Institute of Genetics and Molecular Medicine, The University of Edin- \\ burgh, Crewe Road South, Edinburgh EH4 2XR, UK
}

Ponatinib, a clinically approved tyrosine kinase inhibitor used to treat chronic myeloid leukemia (CML), has an alkyne moiety in its structure that makes it inherently Raman active in the cellular silent region of the Raman spectrum. Stimulated Raman Scattering (SRS) microscopy is a quantitative imaging tool that allows spatial and temporal measurements and discrete intracellular registration by combining with fluorescent reporters. In addition, SRS allows quantitative measurements of intracellular drug concentrations. This has enabled utilisation of SRS microscopy to image label-free ponatinib with high sensitivity and specificity in a number of live human CML cell lines. Drug resistance is a widespread problem in CML treatment, where ponatinib resistant patients have very limited treatment options. To better understand ponatinib resistance mechanisms, a human CML cell line with acquired ponatinib resistance was developed. Ponatinib concentration in parental and ponatinib resistant CML cells was determined and the values were found to be comparable to those obtained by quantitative LC-MS. It was found that lysosomal trapping causes a 730 -fold increase in drug concentration inside the cell. These studies also provide evidence that ponatinib accumulation in lysosomes is a potential CML resistance mechanism, as the intralysosomal concentration was increased 1.9 -fold in the ponatinib resistant cell line. It was further demonstrated that treatment with chloroquine to increase lysosomal $\mathrm{pH}$, reduces the lysosomal concentration of ponatinib by 2 -fold and 2.6-fold in parental and ponatinib resistant cells respectively. This preliminary data suggest that chloroquine combination treatment could be useful in ponatinib resistant CML.

\section{Resistance to chemotherapy used for early and advanced settings in patients diagnosed with triple negative breast cancer - the Kent cancer network experience}

Vasileios Angelis, Emily Parsons, Claire Ryan, Catherine Harper-Wynne

Kent Cancer Network, Maidstone and Tunbridge Wells NHS Trust, Hermitage Lane, Maidstone, Kent ME16 $9 Q Q, U K$ 
Aim: Triple negative breast cancer (TNBC) is an aggressive subtype of breast cancer that is associated with poor prognosis. Due to the lack of hormone sensitivity and the significant heterogeneity amongst triple negative breast cancer there are no approved targeted therapies and cytotoxic chemotherapy remains as the standard treatment. Although response rates to chemotherapy in early disease are high, patients with TNBC remain at high risk of relapse and prognosis remains poor in comparison to other types of breast cancer. There is currently lack of data on this subpopulation of patients with regards to the resistance to chemotherapy used for early disease (primary resistance) and advanced disease (secondary resistance) through patients' clinical treatment pathway.

Methods: Data were collected retrospectively from 163 patients diagnosed with triple negative cancer in Maidstone and Tunbridge Wells Hospitals between 2011-2017. Clinico-pathological characteristics and patient treatment outcomes were obtained by accessing electronic records.

Results: One hundred and sixty-three patients with TNBC were identified with a median age of 57. Of the patients who received treatment for early disease, $50 \%$ of the relapses occurred within 12 months of this treatment (i.e., primary resistance). 33 patients had metastatic disease, 13 of whom received 1st line palliative chemotherapy. Over 50\% (6) of patients experienced disease progression in less than 3 months from commencing treatment (often termed secondary resistance). Patients who subsequently received 2 nd and 3rd line chemotherapy experienced disease progression in less than 1.5 month.

Conclusion: Rates of disease recurrence are consistent with the literature and early progression on treatments exemplifies the inherent chemo-resistance with this phenotype. Continued basic scientific investigation is needed to examine resistance and develop targeted treatments for this poor prognosis disease.

\title{
64. Investigating resistance mechanisms to tamoxifen in oestrogen-receptor positive breast cancer
}

\author{
Joanna L. Bird ${ }^{1}$, Jindrich Cinatl ${ }^{2}$, Mark Wass $^{1}$, Martin Michaelis $^{1}$ \\ ${ }^{1}$ University of Kent, School of Biosciences. Canterbury, Kent, UK \\ ${ }^{2}$ Institute of Medical Virology, Clinics of the Goethe-University, Frankfurt am Main, Germany
}

Tamoxifen is the most commonly used adjuvant therapy used to treat oestrogen receptor (ER) positive breast cancer. However, development of resistance to this drug during treatment regimens presents a serious clinical problem. Over the last 20 years, many advances have been made into understanding the mechanisms of oestrogen signalling in cancer, and the biology of oestrogen receptors, which helps us predict how resistance to this drug arises. However, not everything is understood about the mode of action of tamoxifen as it is known to have both agonistic and antagonistic properties dependent on tissue type. In recent years, it has been increasingly reported that tamoxifen does not just target ERs, although the vast majority of its actions are still considered to be facilitated by these receptors. It is thought to also elicit nonspecific actions in vivo, which ultimately alters the scientific community's perspective of this familiar drug. This study introduces a new panel of tamoxifen resistant ER+ breast cancer cell lines. The cell lines have been characterised for ER expression, response to endogenous oestrogen, and sensitivity to a range of anticancer agents.

\section{The efficacy of doxorubicin-loaded nanoparticles in drug-resistant cancer cell lines}

\author{
Hannah Onafuye ${ }^{1}$, Stefan Pieper ${ }^{2}$, Jindrich Cinatl $\mathrm{Jr}^{3}$, Mark N. Wass ${ }^{1}$, Martin Michaelis ${ }^{1}$ \\ ${ }^{1}$ University of Kent, School of Biosciences, Canterbury, Kent, UK \\ ${ }^{2}$ Institute of Pharmaceutical Technology and Biopharmacy, University of Münster, Münster, Germany
}


${ }^{3}$ Institute of Medical Virology, Clinics of the Goethe-University, Frankfurt am Main, Germany

Drug resistance is the most common cause of failure of anti-cancer drug therapies. Nanoparticles have been introduced as drug carrier systems for the targeting of tumours and overcoming drug resistance. Here, we investigate the sensitivity of cancer cells to doxorubin-loaded nanoparticles made from different materials, using a panel of neuroblastoma cell lines and their doxorubicin and vincristine sub-lines.

\section{Characterisation of triple negative breast cancer cell lines adapted to paclitaxel}

\section{Eithaar M. Al-Barwani ${ }^{1}$, Jindrich Cinatl $\mathrm{Jr}^{2}$, Mark N. Wass ${ }^{1}$, Martin Michaelis ${ }^{1}$}

${ }^{1}$ School of Biosciences, University of Kent, Canterbury, UK

${ }^{2}$ Institute of Medical Virology, Clinics of the Goethe-University, Frankfurt am Main, Germany

Triple negative breast cancer (TNBC) is a specific subtype of breast cancer that is negative for the protein expression of the oestrogen receptor, progesterone receptor and Human epidermal growth factor receptor 2. TNBC constitutes $15 \%-25 \%$ of all breast cancers and has been established as being a very aggressive subtype, despite patients having good initial responses to chemotherapy. One of the chemotherapeutic agents most commonly used as a first line of treatment in TNBC is the microtubule-binding agent paclitaxel. Here we introduce a novel panel of TNBC cell lines adapted to paclitaxel including drug sensitivity profiles against a range of cancer agents.

\section{Characterisation of non-small cell lung cancer with epidermal growth factor receptor mutation}

Tharsagini Nanthaprakash ${ }^{1}$, Jindrich Cinatl $\mathrm{Jr}^{2}$, Mark N. Wass ${ }^{1}$, Martin Michaelis ${ }^{1}$

${ }^{1}$ School, of Biosciences, University of Kent, Canterbury, UK

${ }^{2}$ Institute of Medical Virology, Clinics of the Goethe-University, Frankfurt am Main, Germany

Non-small cell lung cancer (NSCLC) is one of the leading causes of cancer-related deaths and is the most common type of lung cancer (87\%) in the UK. Approximately $70 \%$ of the patients present with locally advanced or metastatic disease at the time of the diagnosis left with chemotherapy as the most beneficial option. NSCLCs harbouring tumour cells characterised by activating epidermal growth factor receptor (EGFR) mutations are typically treated using EGFR tyrosine kinase inhibitors (TKIs). However, resistance formation is inevitable and multiple mechanisms of TKIs resistance have been reported in EGFR-mutant NSCLC. Here, we introduce a novel panel of models of acquired EGFR-TKI resistance in NSCLC based on the EGFR-mutant NSCLC cell lines HCC4006 and HCC827 and their sub-lines adapted to the first generation EGFR-TKIs Erlotinib and Gefitinib and the second generation EGFR-TKI Afatinib. 